\title{
The Sivashinsky equation for corrugated flames in the large-wrinkle limit
}

\author{
Guy Joulin $1, *$ and Bruno Denet ${ }^{2,+}$ \\ ${ }^{1}$ Laboratoire de Combustion et de Détonique, \\ UPR 9028 du CNRS, ENSMA, 1 rue Clément Ader, \\ B.P. 40109, 86961 Futuroscope Cedex, Poitiers, France. \\ ${ }^{2}$ Institut de Recherche sur les Phénomènes Hors d'Equilibre, \\ UMR 6594 du CNRS, Technopole de Château Gombert, \\ 49 rue Joliot-Curie, 13384 Marseille Cedex 13, France.
}

(Dated: October 2, 2018)

\begin{abstract}
Sivashinsky's (1977) nonlinear integro-differential equation for the shape of corrugated 1dimensional flames is ultimately reducible to a $2 N$-body problem, involving the $2 N$ complex poles of the flame slope. Thual, Frisch \& Henon (1985) derived singular linear integral equations for the pole density in the limit of large steady wrinkles $(N \gg 1)$, which they solved exactly for monocoalesced periodic fronts of highest amplitude of wrinkling and approximately otherwise. Here we solve those analytically for isolated crests, next for monocoalesced then bicoalesced periodic flame patterns, whatever the (large-) amplitudes involved. We compare the analytically predicted pole densities and flame shapes to numerical results deduced from the pole-decomposition approach. Good agreement is obtained, even for moderately large Ns. The results are extended to give hints as to the dynamics of supplementary poles. Open problems are evoked.

PACS numbers: 47.20.Ky, 47.54.-r, 47.70.Fw, 82.40.Ck
\end{abstract}

${ }^{*}$ Corresponding author; Electronic address: joulin@lcd.ensma.fr

${ }^{\dagger}$ Electronic address: bruno.denet@irphe.univ-mrs.fr 


\section{INTRODUCTION}

Being able to describe the nonlinear development of the Landau-Darrieus [1, 2] (LD) instability of premixed-flame fronts is a central topic in combustion theory. As early as 1977 Sivashinsky [3] showed, in the limit $\mathcal{A} \ll 1$ of small Attwood numbers based upon the fresh gas $\left(\rho_{u}\right)$ or burnt gas $\left(\rho_{b}<\rho_{u}\right)$ densities, $0<\mathcal{A} \equiv\left(\rho_{u}-\rho_{b}\right) /\left(\rho_{u}+\rho_{b}\right)<1$, that the shape $\phi(x, t)$ of a flat-on-average, spontaneously evolving wrinkled flame is governed by

$$
\phi_{t}+\frac{1}{2} \phi_{x}^{2}=\nu \phi_{x x}+I(\phi)
$$

in suitable units. In (11) the subscripts denote partial derivatives with respect to time, $t$, and coordinate, $x$, normal to the mean direction of propagation, and the "viscosity" $\nu>0$ represents a reciprocal Peclet number based upon the actual flame thickness and the wrinkle wavelength. The linear integral operator $I(\cdot)$ is defined by $I\left(e^{i k x}\right)=|k| e^{i k x}$ (whence $I(\phi)$ is the Hilbert transform, $\hat{H}\left(-\phi_{x}\right)$, of $\left.-\phi_{x}\right)$ and stems from the LD instability. The growth/decay rate of infinitesimal harmonics is $|k|-\nu k^{2}$, which identifies $1 / \nu$ and $\nu$ as neutral wavenumber and minimum growth time, respectively. The nonlinearity is geometrical, accounting as it does for the cosine, $\left(1+s^{2}\right)^{-1 / 2} \simeq 1-s^{2} / 2+\ldots$, of the small angle $(\arctan (s) \simeq s+\ldots)$ that the local normal to the flame front makes to the mean direction of propagation, where $s \sim \phi_{x} \times \mathcal{A}$ is the unscaled front slope. Originally derived in [3] as a leading order result for $\mathcal{A} \rightarrow 0^{+}$, equation (11) happens to govern the shape of steadily propagating fronts even when two more terms of the $\mathcal{A}$-expansion are retained [4, 5]; its structure then remains valid practically up to $\mathcal{A}=3 / 4$, i.e., $\rho_{u}=7 \rho_{b}$ [4] ]

Numerics [6] reveals that "steady" solutions of (1), corresponding to $\phi(x, t)=-V t+\phi(x)$ are often ultimately reached. When (11) is integrated with periodic boundary conditions for "not-too-small" values of $\nu, \nu>1 / 25$ say, the "steady" pattern has a single crest per $x$ wise interval of $2 \pi$ length, where $\phi_{x x}$ is large and negative; without loss of generality one may assume that one is located at $x=0$, in which case $\phi_{x}=0$ when $x$ is an integer multiple of $\pi$ (i.e., $x=0(\bmod \pi))$ and $\phi_{x x}( \pm \pi) \simeq 1 / \pi$. If Neumann conditions at $x=0$ and $x=\pi$ are used instead, still with a moderately small $\nu$, the final pattern obtained from numerical (pseudo-spectral) integrations of (1) may also have an extra crest located at $x=\pi[7]$, with $\phi_{x x}(\pi)$ large and negative. By the very way they are obtained as final state

of an unsteady process the 2-crested patterns have a finite basin of attraction, contrary to the case of periodic boundary conditions [7] where the only stable patterns have a single 
crest per cell; yet such "half-channel" solutions happen to coincide with the restriction to $0 \leq x \leq \pi$ of properly shifted $2 \pi$-periodic ones, for these are symmetric about $x=0$ and $x=\pi$. If $\nu$ is too small the widest patterns get very sensitive to noise, even when caused by numerical rounding-off. In [8] the estimate $\mu \geq O\left(e^{-1 / 2 \nu \kappa}\right) \equiv \mu_{c}(\kappa)$ was obtained for the noise intensity $\mu$ needed to trigger the appearance of extra-cells on top of the main ones with periodic boundary conditions; the number $\kappa$ in the above exponent is $\phi_{x x}( \pm \pi) \simeq 1 / \pi$; since the most rapidly growing noise-induced disturbances (with initial wavenumbers $|k| \simeq 1 / \nu$ [8]) of a nearly parabolic trough undergo an $O\left(e^{1 / 2 \nu \kappa}\right)$ amplification, they ultimately get visible as subwrinkles of $O(1)$ final amplitude if $\mu \geq \mu_{c}(\kappa)$. Having a larger $\phi_{x x}>0$ at their troughs (see Sec. 7), 2-crested patterns are presumably less sensitive to noise than the single-crest ones associated with the same wavelength, because $\mu_{c}(\kappa)$ increases dramatically with $\kappa$ when $\nu$ is small. The numerical work of Ref. [9] also showed that sums $\phi\left(x_{1}, x_{2}, t\right)=\phi_{1}\left(x_{1}, t\right)+\phi_{2}\left(x_{2}, t\right)$ of orthogonal, 2-crested one-dimensional patterns play a central role in the study of (11) generalized to 2-dimensional flames $\left(x \rightarrow\left(x_{1}, x_{2}\right), \phi_{x}^{2} \rightarrow|\nabla \phi|^{2}\right.$, $\phi_{x x} \rightarrow \Delta \phi, I(\cdot) \equiv$ multiplication by $(\boldsymbol{k} \cdot \boldsymbol{k})^{1 / 2}$ in the 2 -D Fourier space $\left.\boldsymbol{k}=\left(k_{1}, k_{2}\right)\right)$ and to rectangular domains in the Cartesian $\left(x_{1}, x_{2}\right)$ plane. Without noise such sums are exact stable solutions; with random additive forcing they recurrently appear as long-lived transients when Neumann conditions are adopted.

Further analyses on the stability of solutions of Eq. (1) and their responses thus seem warranted, and getting the "steady" patterns that correspond to wide, hence large, cells (or small $\nu \mathrm{s}$ ) is a prerequisite. The present contribution is intended to do this.

It is organised as follows. Section 2 introduces the pole-decomposition method, the discrete equations for the pole locations, and the two integral equations that approximate them for large front wrinkles. The latter equations are next solved analytically for isolated crests (Sec. 3) then one-crested periodic patterns (Section 4), and the prediction compared to numerical results from the pole-decomposition approach. Sections 5 and 6 compute the flame speed from the density, and take up the dynamics of a few extra-poles, respectively. Section 7 generalizes the above integral equations to a pair of coupled ones corresponding to 2-crest periodic flames (and "half-channel" ones), then solves them analytically; comparisons with numerics are again presented. We end up with concluding remarks and open problems (Sec. 8). 


\section{POLE-DECOMPOSITION(S)}

In 1985 Thual, Frisch and Henon [10] (herein referred to as "TFH") discovered (see also [11]) that (11) possesses solutions $\phi(x, t)$ representing $2 \pi$-periodic flame patterns with slopes $\phi_{x}$ in the form

$$
\phi_{x}(x, t)=-\nu \sum_{\alpha=-N}^{N} \cot \left(\frac{x-z_{\alpha}}{2}\right),
$$

in which the complex-valued poles of $\phi_{x}(x, t), z_{\alpha}(t)$, are involved in conjugate pairs $\left(z_{-\alpha}=\right.$ $\left.z_{\alpha}^{*}, \alpha \neq 0\right)$ for $\phi_{x}(x, t)$ to be real when $x$ is. For this pole-decomposed expression to solve (1), the $z_{\alpha}^{\prime} \mathrm{s}(\alpha=-N, \ldots,-1,1, \ldots, N)$ must evolve according to the $2 N$-body problem

$$
\frac{d z_{\alpha}}{d t}=\nu \sum_{\substack{\beta=-N \\ \beta \neq \alpha}}^{N} \cot \left(\frac{z_{\beta}-z_{\alpha}}{2}\right)-i \operatorname{sign}\left(\mathcal{I} m\left(z_{\alpha}\right)\right),
$$

where $\operatorname{Im}(\cdot)$ denotes the imaginary parts of $(\cdot)$ and the signum function (with sign $(0)=0)$ accounts for the LD instability. Once (3) is solved for the pole locations, $\phi(x, t)$ is available from (21) and the wrinkling-induced excess propagation speed $V=-\left\langle\phi_{t}\right\rangle>0$ follows from (1):

$$
V=\frac{1}{2}\left\langle\phi_{x}^{2}\right\rangle
$$

where $\langle\cdot\rangle$ stands for an average along the $x$-coordinate; thus, $V$ simply measures the wrinkling-induced fractional increase in flame arclength, since $\left\langle\left(1+s^{2}\right)^{1 / 2}-1\right\rangle=\left\langle s^{2} / 2\right\rangle+\ldots \sim$ $\mathcal{A}^{2} \times V$. Beside periodic $\phi(x, t) \mathrm{s}$, (11) also allows [10] for isolated non periodic wrinkles that have an infinite wavelength, $V=0, \cot (z)$ replaced by $1 / z$, and

$$
\frac{d z_{\alpha}}{d t}=\nu \sum_{\substack{\beta=-N \\ \beta \neq \alpha}}^{N} \frac{2}{z_{\beta}-z_{\alpha}}-i \operatorname{sign}\left(\mathcal{I} m\left(z_{\alpha}\right)\right) .
$$

In the latter situation, the precise value of $\nu>0$ does not matter since it could be scaled out, and the integer $N \geq 1$ is arbitrary. As for (21) (31), the maximum allowed value $N_{\text {opt }}(\nu)$ of $N$ in steady configurations increases with $1 / \nu>1[10]$. As shown by TFH, steady flames obtained from (3) or (5) correspond to poles that "coalesce" (or align) along parallels to the imaginary axis, as a result of the pairwise pole interactions that are attractive along the real $x$-axis and repulsive in the normal direction. In the case of an isolated crest located at $x=0$, the poles ultimately involved in steady solution are of the form $i B_{\alpha},-N \leq \alpha \leq N$, 
$\alpha \neq 0$, with real $B_{\alpha}$ s satisfying coupled discrete equations deduced from (5):

$$
\nu \sum_{\substack{\beta=-N \\ \beta \neq \alpha}}^{N} \frac{2}{B_{\alpha}-B_{\beta}}=\operatorname{sign}\left(B_{\alpha}\right) .
$$

The authors of Ref. [10] also evidenced that the larger the number $N$ of pole-pairs in such "vertical" steady alignments, the smoother the involved poles are distributed along the $B$ coordinate, with $B_{\alpha+1}-B_{\alpha}$ well smaller than $B_{N}$. This suggested TFH to replace the discrete sum in (6) (or its analogue deduced from (3) ) by an integral over the continuous variable $B$, with such a continuous measure that $P(B) d B$ is the number of poles located between $B$ and $B+d B$; a constructive definition of $P(B)$ is specified in (20). In this continuous approximation the steady versions of (2) (3) are amenable to singular Fredholm integral equations, specifically:

$$
f \frac{2 \nu P\left(B^{\prime}\right)}{B-B^{\prime}} d B^{\prime}=\operatorname{sign}(B)
$$

in the non-periodic situations (an isolated wrinkle at $x=0$ ), and

$$
f \nu P\left(B^{\prime}\right) \operatorname{coth}\left(\frac{B-B^{\prime}}{2}\right) d B^{\prime}=\operatorname{sign}(B)
$$

for the monocoalesced $2 \pi$-periodic cases (one single crest per cell, at $x=0(\bmod 2 \pi))$. In (7) (8) $B$ denotes the pole imaginary coordinate, and the Cauchy principal parts $f \cdot d B^{\prime}$ stem from the condition $\beta \neq \alpha$ on the sums featured in (3) (5). Consistent with their interpretation as pole densities, the $P(B) \mathrm{s}$ showing up in (77) (8) both are non-negative even functions of their argument (for $\phi_{x}$ to be real when $x$ is) and are normalized by

$$
\int P\left(B^{\prime}\right) d B^{\prime}=2 N \text {. }
$$

In (77)-(9) the integrals extend over the ranges (to be determined as part of the solutions) where $P(B) \neq 0$. The next sections will solve (7) (8) (9) analytically, starting with the simpler equation (7).

\section{ISOLATED CREST}

Because isolated crests have $\phi_{x} \rightarrow 0$ at $|x| \rightarrow \infty$, we firstly anticipate the existence of some finite $B_{\max }>0$ such that $P\left(|B|>B_{\max }\right) \equiv 0$ in (7). We next recall the identity

$$
\int_{-\pi / 2}^{\pi / 2} \frac{\cos \left((2 M+1) \Phi^{\prime}\right) \cos \Phi^{\prime}}{\sin \Phi-\sin \Phi^{\prime}} d \Phi^{\prime}=\pi \sin ((2 M+1) \Phi)
$$


that can be deduced, through the change of variable $\Phi \rightarrow \Phi+\pi / 2$, from a similar one appearing in the Prandtl theory of lifting lines [12, 13]. Identity (10) allows one to solve such singular integral equations as Wigner's [14] (for the density, $2 \nu P$ say, of eigenvalues of large real random matrices in the Gaussian Orthogonal Ensemble), written here as

$$
f \frac{2 \nu P\left(B^{\prime}\right)}{B-B^{\prime}} d B^{\prime}=B
$$

its solution is the celebrated semi-circle law $2 \pi \nu P(B)=\max \left(B_{\max } \cos \Phi, 0\right)$, [14], provided that one sets

$$
B=B_{\max } \sin \Phi, \quad-\frac{\pi}{2} \leq \Phi \leq \frac{\pi}{2}
$$

in (11). Interestingly, the same change of independent variable in (7) produces

$$
f_{-\pi / 2}^{\pi / 2} \frac{2 \nu P\left(\Phi^{\prime}\right) \cos \Phi^{\prime}}{\sin \Phi-\sin \Phi^{\prime}} d \Phi^{\prime}=\operatorname{sign}(\Phi)
$$

since $\operatorname{sign}(B)=\operatorname{sign}(\Phi)$ for $|\Phi|<\pi$. Over the same range (and hence over the narrower support of $P,|\Phi| \leq \pi / 2$ ), the right-hand side of (13) may be expanded as the Fourier series

$$
\operatorname{sign}(\Phi)=\frac{4}{\pi} \sum_{M=0}^{\infty} \frac{1}{2 M+1} \sin ((2 M+1) \Phi),
$$

consistent with our convention that $\operatorname{sign}(0)=0$. From (10) the solution to (13) can thus be written as a Fourier series of cosines that all vanish at $\Phi= \pm \pi / 2$ :

$$
\begin{aligned}
2 \nu P(\Phi) & =\frac{4}{\pi^{2}} \sum_{M=0}^{\infty} \frac{1}{2 M+1} \cos ((2 M+1) \Phi) \\
& =\frac{1}{\pi^{2}} \log \left(\frac{1+\cos \Phi}{1-\cos \Phi}\right) \\
& =\frac{1}{\pi^{2}} \log \left(\frac{1+\sqrt{1-B^{2} / B_{\max }^{2}}}{1-\sqrt{1-B^{2} / B_{\max }^{2}}}\right)
\end{aligned}
$$

and $P \equiv 0$ for $|B|>B_{\max }$; to get (17) from (16), (12) was explicitly employed.

The cumulative pole distribution $R(B)=\int_{0}^{B} P\left(B^{\prime}\right) d B^{\prime}$ reads, after integration by parts, as

$$
2 \nu R(B)=\frac{B_{\max }}{\pi^{2}}\left(\sin \Phi \log \frac{1+\cos \Phi}{1-\cos \Phi}+2 \Phi\right),
$$

whereby the renormalization condition $R\left(B_{\max }\right)=R(\Phi=\pi / 2)=N$ fixes $B_{\max }$ to be given by

$$
B_{\max }=2 \pi N \nu
$$


TFH [10] fitted the cumulative distribution they obtained from a numerical resolution of (5) for steady arrangements of aligned poles, by the expression $\pi^{2} \nu R=$ $\int_{0}^{B} \log \left(1.28 N \nu \pi^{2} /\left|B^{\prime}\right|\right) d B^{\prime}$ when $|B| \leq B_{\max }$ [10]. Equations (17) (19) show that 1.28 estimated from their numerical pole distribution at $|B| \ll B_{\max }$ actually was a numerical approximation of $4 / \pi=1.273 \ldots$ Figures 1 and 2 compare the analytical findings (18) (19) to our own resolutions of (5), with $N=10$, and 100, respectively. The TFH fit is also displayed for illustration. The pole density $P$ is defined for $\alpha \geq 1$ by

$$
P\left(\left(B_{\alpha}+B_{\alpha-1}\right) / 2\right) \equiv\left(B_{\alpha}-B_{\alpha-1}\right)^{-1}
$$

in terms of the pole locations (with $B_{0}=0$ by convention); it is shown in Fig. 3 for $N=100$, and compared with the continuous approximation (17) and the TFH fit. Once the cumulative distribution is determined by (18) (19) in the continuous limit, approximations $\tilde{B}_{\alpha}$ to the discrete pole locations can be retrieved upon solving [10]

$$
R\left(\tilde{B}_{\alpha}\right)=\alpha-1 / 2, \quad \alpha=1, \ldots, N
$$

numerically (e.g., by the Newton-Raphson method, with the "exact" $B_{\alpha}$ s as initial guess!). The resulting crest shape

$$
\tilde{\phi}(x)=-2 \nu \sum_{\alpha=1}^{N} \log \left(1+\frac{x^{2}}{\tilde{B}_{\alpha}^{2}}\right)
$$

is compared to the exact one (numerical) in Fig. 4 and to that issued from the continuous approximation. The latter profile has

$$
\begin{aligned}
\phi_{x} & =-\int_{-B_{\max }}^{B_{\max }} \frac{2 \nu P(B) d B}{x-i B} \\
& =-\frac{1}{\pi} \operatorname{sign}(x) \log \left(\frac{\sqrt{x^{2} / B_{\max }^{2}+1}+1}{\sqrt{x^{2} / B_{\max }^{2}+1}-1}\right),
\end{aligned}
$$

the second expression resulting from substitution of (17) in (23), then a lucky look at p. 591 of Ref.[15].

As suggested by the form of (23), and confirmed by (24), $\phi_{x}(x)$ is most simply deduced from $P( \pm i x)$ through contour integration in the complex $B$-plane. A further integration by parts of (24) yields the continuous-approximation prediction for $\phi(x)$ (up to an additive constant):

$$
\phi(x)=-\frac{1}{\pi} \operatorname{sign}(x) B_{\max }\left(\sinh \xi \log \frac{\cosh \xi+1}{\cosh \xi-1}+2 \xi\right),
$$


where $x=B_{\max } \sinh \xi$ (compare to (12)). The integration constant was selected in Fig. 4 to achieve good agreement with the exact $\phi(x)$ for $|x| \rightarrow \infty$. Two final remarks: (i) $\nu$ disappeared as a factor in (24) as it should, because $\nu$ can be scaled out; (ii) $\phi(x)$ is of the form $\nu N F(x / \nu N)$, and this scale-invariance shows that the continuous approximation actually amounts to describing $\phi(x)$ at large distances compared to the actual radius of

curvature $\left(1 / \int_{\tilde{B}_{1}}^{B_{\max }} 4 \nu P(B) d B / B^{2}=o(\nu)\right)$ of the flame tip, when $N \gg 1$ ( that is, for large wrinkles).

\section{MONO-COALESCED, PERIODIC CREST}

The following simple remark will allow us to solve (8), i.e., in the case where all the poles of $\phi_{x}$ are aligned along the imaginary $x$-axis $(\bmod 2 \pi)$. Because $P\left(B^{\prime}\right)$ still is an even function of $B^{\prime}$, only the even parts (at fixed $\left.B\right)$ of $\operatorname{coth}\left(\left(B-B^{\prime}\right) / 2\right.$ ) will actually contribute to the integral over $B^{\prime}$. Equation (8) may thus be re-written as

$$
f_{-B_{\max }}^{B_{\max }} \frac{\nu P\left(B^{\prime}\right)\left(1-\tanh ^{2}\left(B^{\prime} / 2\right)\right)}{\tanh (B / 2)-\tanh \left(B^{\prime} / 2\right)} d B^{\prime}=\operatorname{sign}(B),
$$

upon use of the known formula for the tanh $(\cdot)$ of a difference, and neglect of a term proportional to $f P\left(B^{\prime}\right) \tanh \left(B^{\prime} / 2\right) d B^{\prime}=0$. We now set

$$
\tanh \left(\frac{B}{2}\right)=\tanh \left(\frac{B_{\max }}{2}\right) \sin \Phi, \quad-\frac{\pi}{2} \leq \Phi \leq \frac{\pi}{2},
$$

converting (26) into

$$
\int_{-\pi / 2}^{\pi / 2} \frac{2 \nu P\left(B^{\prime}\right) \cos \Phi^{\prime}}{\sin \Phi-\sin \Phi^{\prime}} d \Phi^{\prime}=\operatorname{sign}(\Phi),
$$

which is nothing but (13). Therefore the sought after pole-density is still given by (16), the only difference with the previous non periodic case being that $B, B_{\text {max }}$, and $\Phi$ are now related by (27) instead of (12).

The new cumulative density $R(B)=\int_{0}^{B} P\left(B^{\prime}\right) d B^{\prime}$ is given, after an integration by parts, by

$$
\begin{aligned}
\pi^{2} \nu R(B)= & \frac{1}{2} \log \frac{1+A \sin \Phi}{1-A \sin \Phi} \log \frac{1+\cos \Phi}{1-\cos \Phi} \\
& +\int_{0}^{\Phi} \log \frac{1+A \sin \Phi^{\prime}}{1-A \sin \Phi^{\prime}} \frac{d \Phi^{\prime}}{\sin \Phi^{\prime}}
\end{aligned}
$$


$A \equiv \tanh \left(B_{\max } / 2\right)$, whereby the normalization (9) requires

$$
N \nu \pi^{2}=\int_{0}^{\pi / 2} \log \frac{1+A \sin \Phi}{1-A \sin \Phi} \frac{d \Phi}{\sin \Phi} .
$$

As the above integral turns out to be $\pi \arcsin A$ (p. 591 of [15]) the range of $P(B)$, still given by $R\left(B_{\max }\right)=N$, now satisfies

$$
\tanh \left(B_{\max } / 2\right)=\sin (\pi N \nu)
$$

instead of (19). The latter and (31) coincide for $\pi N \nu \ll 1$, as do the associated pole densities. The maximum $B_{\max }$ allowed by (31), $B_{\max }=+\infty$, has $2 N \nu=1$ and $\cos \Phi \equiv 1 / \cosh (B / 2)$, whence $P(B)$ resumes the form

$$
P(B)=\frac{1}{\pi^{2} \nu} \log \left(\operatorname{coth} \frac{|B|}{4}\right)
$$

obtained by TFH via Fourier transformations. The figure 5 compares our predictions (29) and (31) with very accurate solutions of (3) for $N=100$ and $2 N \nu=1$. Very good agreement is obtained even if $N$ is only moderately large, and carries over to the pole densities themselves. Again, approximate solutions $\tilde{B}_{\alpha}$ can be retrieved from the analogue of (21), and an approximate flame front shape from

$$
\tilde{\phi}(x)=-2 \nu \sum_{\alpha=1}^{N} \log \left(1-\cos x \operatorname{sech} \tilde{B}_{\alpha}\right)+\text { const. }
$$

Figure [6] shows of a comparison between (33), the exact flame shape obtained from the exact (yet obtained numerically) $B_{\alpha}$ s satisfying (3), and the curve deduced from the continuous approximation, for which the flame slope $\phi_{x}(x)$ reads

$$
\phi_{x}=-\nu \int \cot \left(\frac{x-i B}{2}\right) P(B) d B,
$$

again a real function because $P(-B)=P(B)$. With $P(B)$ given by (16) (27) (31) the above integral can be reduced to one available in p. 591 of [15] and yields (for $-\pi \leq x \leq \pi$ ):

$$
\phi_{x}(x)=-\frac{1}{\pi} \operatorname{sign}(\xi) \log \frac{\cosh \xi+1}{\cosh \xi-1}, \quad \tan \frac{x}{2} \equiv A \sinh \xi
$$

thereby confirming that $\phi_{x}(x)$ is accessible from $P(B)$ by analytical continuation to $\pm i x$. In particular, the TFH solution, eq. (32), has $\pi \phi_{x}=-2 \operatorname{sign}(x) \log |\cot x / 4|$ and $\phi_{x x}( \pm \pi)=$ $1 / \pi$; more generally, $\phi_{x x}( \pm \pi)=A / \pi$. A further integration by parts yields

$$
\begin{aligned}
-i \pi \phi(x)= & \operatorname{sign}(x) \log \frac{1+i A \sinh \xi}{1-i A \sinh \xi} \log \frac{\cosh \xi+1}{\cosh \xi-1} \\
& +2 \operatorname{sign}(x) \int_{0}^{\xi} \log \frac{1+i A \sinh \xi^{\prime}}{1-i A \sinh \xi^{\prime}} \frac{d \xi^{\prime}}{\sinh \xi^{\prime}},
\end{aligned}
$$


which cannot be evaluated in simple closed form, but may be compared to (29); of course $\phi(x)$ is real when $x$ is, since the complex $\log (\cdot)$ in (36i) also reads $2 i \arctan (A \sinh \xi)=i x$. Note that $\phi(x)$ has the form $F(x ; N \nu)$, in the present units where the pattern is $2 \pi$-periodic. Adopting $\Lambda \neq 2 \pi$ as wavelength would give $2 \pi \phi=\Lambda F(2 \pi x / \Lambda ; 2 \pi N \nu / \Lambda)$ with the same $F$. Accordingly, if $\nu N / \Lambda$ is kept fixed, $\phi_{x x}( \pm \Lambda / 2)$ scales like $1 / \Lambda$ as it should for $\nu \rightarrow 0$, whereby halving the wavelength renders the patterns less sensitive to noise (see the Introduction).

\section{FLAME SPEED FROM CONTINUOUS POLE-DENSITY}

Plugging (34) into (4) allows the wrinkling-induced increase in flame speed $V$ to be written as

$$
2 V=\nu^{2} \iint P(B) P\left(B^{\prime}\right)\left\langle\cot \frac{x-i B}{2} \cot \frac{x-i B^{\prime}}{2}\right\rangle d B d B^{\prime} .
$$

Although the one-variable integrals involved when squaring (34) are ordinary ones, they may be written as principal parts. We next invoke the trigonometric identity $\cot a \cot b=$ $-1+\cot (a-b)(\cot b-\cot a)$ and the average

$$
\left\langle\cot \frac{x-i B}{2}\right\rangle=i \operatorname{sign}(B)
$$

to transform (37) into

$$
\begin{gathered}
2 \frac{V}{\nu^{2}}=-\iint P(B) P\left(B^{\prime}\right) d B d B^{\prime} \\
+2 \int f \operatorname{sign}(B) P(B) P\left(B^{\prime}\right) \operatorname{coth}\left(\frac{B-B^{\prime}}{2}\right) d B^{\prime} d B
\end{gathered}
$$

The first double integral $\left(=\left(\int P(B) d B\right)^{2}\right)$ in (39) follows from the normalization (9), and is $(2 N)^{2}$. The second one is obtained from (8) after multiplication of both sides by $P(B) \operatorname{sign}(B) d B$ and subsequent integration over $B$ : by (9), it is $2 N / \nu$. Thus the simple formula

$$
V=2 N \nu(1-N \nu)
$$

ensues; notice that it was obtained without having to solve (8). Actually (39) can be shown from (3) to hold whatever $N$ and $\nu$ [16], again without solving the pole-equations themselves.

In view of the accuracy of (40) one may inquire whether the solutions of (77) (8) satisfy the "inviscid" Sivashinsky equation, i.e. (1) with $\nu=0$, in the steady cases. To show they 
do, for $x \neq 0$ at least, one may set $\mathcal{P}=P \nu$ and $\mathcal{N}=N \nu$ to remove $\nu$ from (7) (9), then process the Landau-Darrieus term of (1) as follows in the case of an isolated crest:

$$
\begin{aligned}
2 i I(\phi) & =\int \frac{4 \mathcal{P}(B) \operatorname{sign}(B)}{x-i B} d B \\
& =\int \frac{2 \mathcal{P}(B)}{x-i B} d B f \frac{2 \mathcal{P}\left(B^{\prime}\right)}{B-B^{\prime}} d B^{\prime}+\left(B \leftrightarrow B^{\prime}\right) \\
& =\iint \frac{4 i \mathcal{P}(B) \mathcal{P}\left(B^{\prime}\right)}{(x-i B)\left(x-i B^{\prime}\right)} d B d B^{\prime}=i \phi_{x}^{2},
\end{aligned}
$$

where the notation $\left(B^{\prime} \leftrightarrow B\right)$ represents a second copy of the integral that precedes it, with $B$ and $B^{\prime}$ interchanged. The lines above successively use (7), acknowledge that $\left(B, B^{\prime}\right)$ are dummy variables of integration that may be interchanged, then employ (23) squared. Hence (25) satisfies (11) when $\nu=0$ and $\mathcal{N}$ is prescribed, if $x \neq 0$. Thanks to (39), a similar analysis applies to (18), provided $x \neq 0(\bmod 2 \pi)$.

Beside providing one with an exact $P(B)$, eq. (16) shows that (8) admits a continuum of solutions, for there exists nothing in (9) to tell one that $N$ ought to be an integer; this will be commented later (see Sec. 8). One finally specializes (8) to $B=B_{\max }$ to show that $N$ is constrained by $0 \leq 2 N \nu \leq 1$, $\operatorname{since} \operatorname{coth}\left(B_{\max }-B\right) \geq 1$ (see also (31)).

\section{DYNAMICS OF SUPPLEMENTARY POLE-PAIRS}

In 2000, Vaynblatt \& Matalon [17] addressed the linear stability of pole-decomposed monocoalesced "steady" solutions $-V t+\phi(x)$ to (1). Upon writing $\phi(x, t)+V t-\phi(x) \sim$

$\exp (\omega t) \psi_{\omega}(x) \ll 1$ then analytically solving the linearised dynamics to get $\omega$ and $\psi_{\omega}(x)$, the authors of [17] identified two types of linear modes. The modes of type I describe how the $2 N$ poles of $\phi_{x}(x)$ evolve when displaced by infinitesimal amounts from equilibrium; all those are stable $(\omega<0)$, but one that has $\omega=0$ (see below). The modes of type II were interpreted [17, 18] as resulting from $x$-periodic arrays of poles at $\pm i \infty$ that may spontaneously approach the real axis if $N$ is too small for the selected $\nu<1$. The overall conclusion was thus: when endowed with $2 \pi$-periodic boundary conditions, all the monocoalesced solutions are linearly unstable, except a single one that has $N=N_{\text {opt }}(\nu) \equiv\lfloor(1+1 / \nu) / 2\rfloor \simeq 1 / 2 \nu(\lfloor\cdot\rfloor \equiv$ integer part) and is neutrally stable $(\omega=0)$ against shifts along the $x$-axis, the corresponding antisymmetric eigenmode being $\psi_{0}(x)=\phi_{x}(x)$. For $N<N_{\text {opt }}$, modes of type II can manifest themselves, two particularly dangerous ones corresponding to incipient secondary wrinkles 
centred on the main crests $(x=0, \bmod 2 \pi)$ or troughs $(x=\pi, \bmod 2 \pi)$.

When Neumann conditions are employed instead, the aforementioned shifts are not allowed any longer because $\psi_{0 x} \neq 0$ at $x=0$ and $x=\pi$. Numerical integrations [7] of (1) and (3) evidence that there may then exist stable bi-coalesced patterns comprising an extra crest located at $x=\pi$. Even though the steady $2 \pi$-periodic patterns also satisfy (1) with Neumann conditions when properly shifted to have $\phi_{x}(0)=0=\phi_{x}(\pi)$ no stability analysis similar to [17] is yet available in this case; yet instabilities then necessarily require $N<N_{\text {opt }}(\nu)$. Here we address a restricted aspect of the problem, namely: we study how the previously determined monocoalesced "steady" solutions (25) (36) interact with extra pairs of poles. Since the free dynamics (3) conserves the total number of pole pairs at its $t=0$ value, it makes sense to consider $\phi(x, 0)$ s that involve them in a larger number $(N+n)$ than the $N=O(1 / \nu)$ ones retained in a steady profile $\phi(x)$. Each of the $n$ supplementary pairs at $x_{m}(t) \pm i y_{m}(t)$ contributes a perturbation $\phi_{m}(x, t)=\left\langle\phi_{m}\right\rangle-4 \nu \sum_{j \geq 1} \exp \left(-j\left|y_{m}\right|\right) \cos \left(j\left(x-x_{m}\right)\right) / j$ to the flame shapes (this follows from (2) via a term-by-term Fourier expansion [10]) and, as shown in [18], superposing $\phi_{m} \mathrm{~s}$ can reproduce virtually any disturbance $\phi(x, 0)-\phi(x)$. In the present formulation the only difference between Neumann and $2 \pi$-periodic boundary conditions deals with the initial phases $x_{m}(0)$ : whereas the former require the $x_{m}$ s to be compatible with the $x \leftrightarrow-x$ and $\pi-x \leftrightarrow \pi+x$ symmetries, the latter do not.

Contrary to the more conventional normal-mode method (to which it is equivalent if $\left.\left|y_{m}(0)\right| \gg 1[18]\right)$, the pole approach can follow the disturbances when significant nonlinear effects set in... if one is able to solve the $2 N+2 n$ coupled equations for the pole trajectories in the complex plane. The next remark somewhat simplifies the task. In the limits $N \gg 1$, $\nu \rightarrow 0^{+}$and $\nu N=O(1)$ that led to (8), accounting for $n=O(1)$ extra pole pairs - as is assumed here - exerts only a small $O(\nu)$ perturbation on the $2 N$ poles already aligned. Accordingly the distribution $P(B)$ of poles along the main alignments at $x=0(\bmod 2 \pi)$ may be kept unchanged, and given by (16) (27) (31), when computing the motion of $2 n$ supplementary ones.

In the illustrative examples that follow only two extra poles $(n=1)$ located at $\pm i y(t)(\bmod 2 \pi), y>0$, then at $\pi \pm i y(t)$ are considered, to begin with. 


\section{A. Extra-poles at $\mathrm{x} \simeq 0(\bmod 2 \pi)$}

When the two supplementary poles are located at $\pm i y(t)(\bmod 2 \pi)$, their altitude $y(t)$ is determined from (3) - within $O(\nu, 1 / N)$ fractional errors in the limits $N \gg 1, \nu \rightarrow 0^{+}$and $\nu N=O(1)-$ by the ODE

$$
\begin{aligned}
\frac{d y}{d t} & =f \nu P\left(B^{\prime}\right) \operatorname{coth}\left(\frac{y-B^{\prime}}{2}\right) d B^{\prime}-1 \\
& =\frac{2}{\pi} \arcsin (\sin (\pi N \nu) \operatorname{coth}(y / 2))-1, \quad|y| \geq B_{\max }
\end{aligned}
$$

where $P(B)$ is the same as given by (16) (27) and (31), to leading order, and leads to the closed form (43) on integration [15]; for $|y| \leq B_{\max }, d y / d t=0$ by (8). Therefore, whenever $0<1-2 \nu N=O(1)$ and $\nu \rightarrow 0^{+}$, any initial $y(0)>B_{\max }$ will ultimately lead to $y(+\infty)=B_{\max }^{+}$, thereby adding one new incomer to the already present continuum. Put in words: if $2 \nu N<1$ initially, the main pattern is unstable to disturbances with poles at $\pm i y(t)$, and the latter process tends to make $2 N \nu$ approach 1 from below.

Periodic boundary conditions would allow the supplementary pair to be initially off the $x$-axis, say at $x(0) \pm i y(0)$ with $0<x(0)<\pi(\bmod 2 \pi)$. The "horizontal" attraction by the main pole condensation at $x=0(O(\nu)$, actually) [10] will make $x(t)$ decrease, while $y(t)$ still does if $2 N \nu<1$. Ultimately, the extra pole pair will join the main pole alignment (in finite time), and the previous conclusion is qualitatively unchanged: the process makes $N$ increase by one. When Neumann conditions are adopted, however, at least two pairs $\pm x(t) \pm i y(t)$ are needed if $x(t) \neq 0$, to meet the requirement of symmetry about $x=0$, and two possibilities are encountered as to their fate. In the first instance, corresponding to nottoo-small $x(0)$ s and moderate values of $y(0)$, the process is qualitatively the same as above, except that 2 pairs simultaneously join the main condensation at $|y|<B_{\max }$, thereby making $N$ increase by 2 . If $x(0)$ is small and $y(0)$ well above $B_{\max }$, the horizontal mutual attraction between the pair members may make them hit the $x=0$ axis at such a finite time $t_{c}$ that $y\left(t_{c}\right)>B_{\max } ;$ this is best shown from (3) specialized to $x(t) \ll 1$, whereby $d x / d t \simeq-\nu / x$ then $x^{2}(t)+2 \nu\left(t-t_{c}\right) \simeq 0$ for $t \lesssim t_{c}$. The double pole thus formed at $i y\left(t_{c}\right)$ then instantly splits into two simple ones lying on the $x=0$ axis at $y(t)-y\left(t_{c}\right) \sim \pm\left(t-t_{c}\right)^{1 / 2}$, leading

to a subsequent dynamics that ultimately ends like at the beginning of this subsection if $2 N \nu<1$. 


\section{B. Extra-poles at $\mathrm{x} \simeq \pi(\bmod 2 \pi)$}

In case the supplementary poles are located at $\pi \pm i y(t)$ eq. (42) is replaced by

$$
\frac{d y}{d t}=\frac{2}{\pi} \arcsin (\sin (\pi N \nu) \tanh (y / 2))-1+\nu \operatorname{coth} y,
$$

since $\operatorname{coth}(u+i \pi / 2)=\tanh u$. Even though $\nu \ll 1$ the last term in (44), stemming from the interaction of the extra-pole with its complex conjugate, cannot be simply discarded, for otherwise (44) would not be uniformly valid if $y$ gets small. According to (44), any initial $y(0)$ indeed ultimately leads to $y(+\infty)=\nu+o(\nu)$ and to a small $(O(\nu))$ stable disturbance centred at $x=\pi(\bmod 2 \pi)$ whenever $2 N \nu<1$. Although the main pattern's curvature $\phi_{x x}(\pi)>0$ is $O(1)$, and the $O(\nu)$ contribution to $\phi(x, t)$ of the extra pole-pair is small, it is nevertheless enough [7] to render the flame shape $\phi(x, t)$ concave downward at $x=\pi$; as

shown in [7], this occurs as soon as the extra poles enter a thin strip about the real axis, $|y| \lesssim \sqrt{4 \pi \nu}$. Incorporating $O(N)$ extra pairs will also do, but the process of dynamical trough splitting is not within reach of such ODEs as (44) when $n=O(N)$. The structure of 2-crested steady patterns with $n=O(N)$ will be studied in Sec.VII.

Like in VIA one might begin generalizing the present discussion by envisaging a single pair of extra poles off the $x=\pi$ axis, but this is already covered in the preceding paragraphs: if $0<x(0)<\pi$ the pair ultimately joins the poles at $x=0(\bmod 2 \pi)$. It is more revealing to consider two such pairs at $\pi \pm x(t) \pm i y(t)$ with $x(t)$ "small enough", in a way compatible with Neumann conditions, because something new appears. Comparatively large $x(0)$ s will clearly lead to pairs that ultimately stick at $x=0(\bmod 2 \pi)$, because their mutual horizontal attraction could not oppose that of the main alignments. The other extreme of very small $x(0)$ s again leads to the formation of double poles at some $\pi \pm i y\left(t_{c}\right)$, then a subsequent evolution of the two pairs $\pi \pm i y_{1,2}$ along the line $x=\pi(\bmod 2 \pi)$ until they settle at $O(\nu)$ distances to the real axis if $2 N \nu<1$. The important conclusion is that stable 2-crest patterns exist when Neumann conditions are used and $2 N \nu<1$.

By continuity there exist separating trajectories $S_{ \pm}$, such that none of the above behaviours is observed if the pole pairs initially sit on them. The lines $S_{ \pm}$lead the two supplementary pairs towards an unstable equilibrium, a result of a competition between attraction by the main pole population at $x=0(\bmod 2 \pi)$, and the mutual attractions/repulsions among the pair members. For $\nu \ll 1$, and $N \nu=O(1)$, using the steady version of (3) 
and the pole-density given by (16) (27) (31), one can show that such equilibriums correspond to $x(+\infty)= \pm(2 \pi \nu / A)^{1 / 2}+\cdots$ and $y(+\infty)= \pm \nu+\cdots$ to leading order, again with $A=\tanh \left(B_{\max } / 2\right)=\sin (\pi N \nu)$. This shows that there exist even more general steady so-

lutions than considered elsewhere in the paper and in the literature (except in [7] where a similar conjecture was made on a numerical basis). One could have included other pairs as well, some of which along the $x=\pi(\bmod 2 \pi)$ axis.

Our last remark is to again stress that the free dynamics (3) conserves the total number of poles (if finite). By the same token, allowing this number to vary with time is a means to study a forced version of the Sivashinsky equation: adding a pair of poles $x_{m} \pm i y_{m}$ at $t=t_{m}$ amounts to accounting for a term $\phi_{m}(x) \delta\left(t-t_{m}\right)$ in the right-hand side of (1), and combining many $\phi_{m}$ s with various phases (as to vary their signs), amplitudes $\left(\simeq-4 \nu \exp \left(-\left|y_{m}\right|\right)\right.$ if $\left.\left|y_{m}\right| \gg 1\right)$ and times of implantation $\left(t_{m}\right)$ could help one investigate the response of flames to a rich class of weak random noises. We understand that a similar proposal was developed about the "kicked" Burgers equation [19], i.e., (11) without the integral term in the onedimensional case.

\section{BI-COALESCED PERIODIC PATTERNS}

We now take up the structure of "steady" $2 \pi$-periodic solutions of (1) that would have $N$ pairs of poles $i B_{\alpha}(\bmod 2 \pi), \alpha= \pm 1, \pm 2, \ldots, \pm N$, and $n=O(N)$ other pairs at $\pi+i b_{\gamma}$ $(\bmod 2 \pi), \gamma= \pm 1, \pm 2, \ldots, \pm n$. For brevity, we will say that the pole-alignments reside "at" $x=0$ or $x=\pi$, respectively, like the two crests per-cell they correspond to. Because $\operatorname{coth}(u+i \pi / 2) \equiv \tanh (u)$, the steady versions of (3) corresponding to such bi-coalesced flame patterns read as

$$
\begin{aligned}
& \nu \sum_{\substack{\beta=-N \\
\beta \neq \alpha}}^{N} \operatorname{coth}\left(\frac{B_{\alpha}-B_{\beta}}{2}\right) \\
& \quad+\nu \sum_{\delta=-n}^{n} \tanh \left(\frac{B_{\alpha}-b_{\delta}}{2}\right)=\operatorname{sign}\left(B_{\alpha}\right), \\
& \nu \sum_{\substack{\delta=-n \\
\delta \neq \gamma}}^{n} \operatorname{coth}\left(\frac{b_{\gamma}-b_{\delta}}{2}\right)
\end{aligned}
$$




$$
+\nu \sum_{\beta=-N}^{N} \tanh \left(\frac{b_{\gamma}-B_{\beta}}{2}\right)=\operatorname{sign}\left(b_{\gamma}\right) .
$$

In the distinguished limits $\nu \rightarrow 0^{+}, N \nu=O(1), n \nu=O(1)$, the poles at $x=0$ and $x=\pi$ get densely packed (at the scale of the wavelength), with densities $P(B)$ and $p(b)$, respectively. Both $P$ and $p$ will be nonnegative and, in general, compactly supported: $P\left(|B| \geq B_{\max }\right)=0=p\left(|b| \geq b_{\max }\right)$. The ranges $B_{\max }$ and $b_{\max }$ are to be found as part of the solutions to the continuous versions of (45) and (46):

$$
\begin{aligned}
& f \nu P\left(B^{\prime}\right) \operatorname{coth}\left(\frac{B-B^{\prime}}{2}\right) d B^{\prime} \\
& +\int \nu p\left(b^{\prime}\right) \tanh \left(\frac{B-b^{\prime}}{2}\right) d b^{\prime}=\operatorname{sign}(B), \\
& f \nu p\left(b^{\prime}\right) \operatorname{coth}\left(\frac{b-b^{\prime}}{2}\right) d b^{\prime} \\
& +\int \nu P\left(B^{\prime}\right) \tanh \left(\frac{b-B^{\prime}}{2}\right) d B^{\prime}=\operatorname{sign}(b),
\end{aligned}
$$

that are valid for $|B| \leq B_{\max }$ and $|b| \leq b_{\max }$, respectively. To restore some symmetry we set

$$
\begin{gathered}
\tanh (B / 2)=A \sin \Phi, A \equiv \tanh \left(B_{\max } / 2\right) \leq 1 \\
\tanh (b / 2)=a \sin \varphi, \quad a \equiv \tanh \left(b_{\max } / 2\right) \leq 1
\end{gathered}
$$

in (47) (48), then acknowledge that both $P(\cdot)$ and $p(\cdot)$ are even functions, which allows one to suppress some odd parts of the integrands, viewed as functions of $\Phi^{\prime}$ (or $\varphi^{\prime}$ ) at fixed $\Phi$ (or $\varphi$ ). Some cumbersome algebra ultimately transforms (47) and (48) into

$$
\begin{aligned}
& f \frac{2 \nu P\left(\Phi^{\prime}\right) \cos \Phi^{\prime}}{\sin \Phi-\sin \Phi^{\prime}} d \Phi^{\prime}+A a \sin \Phi \\
& \quad \times \int \frac{2 \nu p\left(\varphi^{\prime}\right) \cos \varphi^{\prime}}{1-A^{2} a^{2} \sin ^{2} \varphi^{\prime} \sin ^{2} \Phi} d \varphi^{\prime}=\operatorname{sign}(\Phi) \\
& \quad f \frac{2 \nu p\left(\varphi^{\prime}\right) \cos \varphi^{\prime}}{\sin \varphi-\sin \varphi^{\prime}} d \varphi^{\prime}+A a \sin \varphi \\
& \quad \times \int \frac{2 \nu P\left(\Phi^{\prime}\right) \cos \Phi^{\prime}}{1-A^{2} a^{2} \sin ^{2} \Phi^{\prime} \sin ^{2} \varphi} d \Phi^{\prime}=\operatorname{sign}(\varphi)
\end{aligned}
$$


where all the variables $\left(\Phi, \Phi^{\prime}\right),\left(\varphi, \varphi^{\prime}\right)$ are now taken in the common $[-\pi / 2, \pi / 2]$ range. One may thus adopt a common notation for them, $\left(\sigma, \sigma^{\prime}\right)$ say, in both (51) and (52) and subtract the results to eliminate the $\operatorname{sign}(\cdot)$ functions in the right-hand sides. This produces an homogeneous equation for the difference $P(\cdot)-p(\cdot)$, of which one obvious solution is $P-p \equiv 0$. Hence the important result: if $P=p$ is indeed a viable solution, then

$$
\begin{gathered}
P(B)=J\left(\sigma=\arcsin \left(\frac{\tanh B / 2}{A}\right)\right), \\
p(b)=J\left(\sigma=\arcsin \left(\frac{\tanh b / 2}{a}\right)\right),
\end{gathered}
$$

where $J(\sigma)$ is the same function for both. The even $J(\cdot)$ function itself is then found from (51) or (52) to satisfy

$$
\begin{aligned}
& f_{-\pi / 2}^{\pi / 2} \frac{2 \nu J\left(\sigma^{\prime}\right) \cos \sigma^{\prime}}{\sin \sigma-\sin \sigma^{\prime}} d \sigma^{\prime}+A a \\
& \times \int_{-\pi / 2}^{\pi / 2} \frac{2 \nu J\left(\sigma^{\prime}\right) \sin \sigma \cos \sigma^{\prime}}{1-A^{2} a^{2} \sin ^{2} \sigma^{\prime} \sin ^{2} \sigma} d \sigma^{\prime}=\operatorname{sign}(\sigma) .
\end{aligned}
$$

Further changing the independent variable to $\theta$, with

$$
\sin \theta=\frac{(1+A a) \sin \sigma}{1+A a \sin ^{2} \sigma},
$$

fortunately converts the seemingly hopeless (55) into a form equivalent to the already solved Eq. (28), $\theta$ playing the part that the former $\Phi$ did there (most easily shown by starting from (28) ). Accordingly, the solution to (55) is available in terms of the already found poledensity pertaining to the isolated crests, then the monocoalesced ones: from (16) one indeed has

$$
2 \nu J(\sigma)=\frac{1}{\pi^{2}} \log \frac{1+\cos \theta}{1-\cos \theta}
$$

with $\theta$ defined in (56). As said earlier, eqs. (53) (54), $P(B)$ is immediately retrieved upon setting $\sin \sigma=\tanh (B / 2) \operatorname{coth}\left(B_{\max } / 2\right)$ in (56) (57); same operation to get $p(b)$ from $J(\sigma)$, upon setting $\sin \sigma=\tanh (b / 2) \operatorname{coth}\left(b_{\max } / 2\right)$ in (57) (56) $)$.

The first step to get $B_{\max }$ and $b_{\max }$ again is to compute the cumulative pole-densities. For example $R(B)=\int_{0}^{B} P\left(B^{\prime}\right) d B^{\prime}$ is computed as follows from (157) (156):

$$
\begin{aligned}
2 \pi^{2} \nu R(B)= & \int_{0}^{\Phi} \log \left(\frac{1+\cos \theta^{\prime}}{1-\cos \theta^{\prime}}\right) \frac{2 A \cos \Phi^{\prime} d \Phi^{\prime}}{1-A^{2} \sin ^{2} \Phi^{\prime}}, \\
= & \log \frac{1+A \sin \Phi}{1-A \sin \Phi} \log \frac{1+\cos \theta}{1-\cos \theta} \\
& +2 \int_{0}^{\theta(\Phi)} \frac{d \theta^{\prime}}{\sin \theta^{\prime}} \log \frac{1+A \sin \Phi^{\prime}}{1-A \sin \Phi^{\prime}}
\end{aligned}
$$


again with the understanding that $\Phi$ (or $\Phi^{\prime}$ ) is viewed as a function of $\theta$ (or $\left.\theta^{\prime}\right)$ via ( $(56)$, and conversely; (58) is obtained from the definition of $R(B)$ upon setting $\tanh (B / 2)=A \sin \Phi$, and (59) results from an integration by parts. The cumulative density pertaining to $p(b)$ is obtained in the same way from (157) (56) $)$, now thanks to $\tanh (b / 2)=a \sin \varphi$ : the result is like (59), except for the substitutions $A \rightarrow a, \Phi \rightarrow \varphi, B \rightarrow b, R(B) \rightarrow r(b)$. The normalisations $R\left(B_{\max }\right)=N$ and $r\left(b_{\max }\right)=n$ thus impose the two conditions

$$
\begin{aligned}
N \nu \pi^{2} & =\int_{0}^{\pi / 2} \frac{d \theta}{\sin \theta} \log \frac{1+A \sin \Phi}{1-A \sin \Phi} \\
n \nu \pi^{2} & =\int_{0}^{\pi / 2} \frac{d \theta}{\sin \theta} \log \frac{1+a \sin \varphi}{1-a \sin \varphi}
\end{aligned}
$$

that may be compared to the former equation (30), and reduce to it when $A a=0$. Although we could not compute the above normalization integrals in closed forms, this can be done numerically without difficulty to get $A$ and $a$ as function of $N \nu$ and $n \nu$ (or conversely). Note that $N \geq n$ is equivalent to $A \geq a$. $N>n$ also implies that $R(\cdot)>r(\cdot)$ when both are evaluated at the same argument, Fig. 8.

Before closing this section, it remains to compare the above predictions to direct numerical resolutions of (45) (46), by the Newton-Raphson method. This is done in Figs 7 and 8 , Figure 7 will hopefully convince the reader that both $P(B)$ and $p(b)$ can be expressed in terms of the single function $J(\sigma)$ given by (57).

Now that the pole densities are available, one may try to compute the corresponding increase in flame speed, $V$, from (47) (48) without solving them (like in Sec. 5), to produce

$$
V=2 \nu(N+n)(1-(N+n) \nu)
$$

this simple formula reduce to (40) if $n=0$, and could have been deduced directly from the discrete pole-equations, without solving them. The sum $N+n$ plays the part $N$ did for monocoalesced patterns and, as is shown upon specializing (47) to $B=B_{\max }$, has to satisfy $2(N+n) \nu \leq 1$.

As mentioned earlier, the flame slope $\phi_{x}(x)$ pertaining to the continuous approximation(s) can be obtained directly from the corresponding pole-density(ies) via an analytical continuation from the real $B$ (or $b$ ) axis to $\pm i x$. Using the same procedure here gives, for 
$0 \leq x \leq \pi$

$$
\begin{aligned}
\phi_{x} & =-\frac{1}{\pi} \operatorname{sign}(\bar{x}-x) \log \frac{\cosh \psi+1}{\cosh \psi-1} \\
\sinh \psi & =\frac{(1+A a) \tan x / 2}{A-a \tan ^{2} x / 2}
\end{aligned}
$$

for bicoalesced flames, $\bar{x}$ being the point where $\sinh ^{2} \psi \rightarrow \infty$ in (64) and, therefore, $\phi_{x}(\bar{x})=$ 0 :

$$
\bar{x}=2 \arctan \sqrt{\frac{\tanh B_{\max } / 2}{\tanh b_{\max } / 2}} .
$$

At the flame front trough, $\kappa=\phi_{x x}(\bar{x})=2(A+a) / \pi(1+A a)>A / \pi$ : the corresponding critical noise amplitude $\mu_{c}(\kappa)$ needed to trigger the appearance of subwrinkles markedly exceeds that pertaining to monocoalesced fronts (see Sec. 1). Two extra pole-pairs initially placed at the points $\pm \bar{x} \pm i \nu(\bmod 2 \pi)$ would stay there in unstable equilibrium. There exist separating trajectories $S_{ \pm}$passing through them, which delineates the basins of attraction of the main pole condensations at $x=0$ or $x=\pi$. Only the trajectories of initially remote extra-poles that are close enough to $S_{ \pm}$will enter the $O(\sqrt{\nu})$ strip adjacent to the $B=0$ axis where their direct influence on the main pattern becomes visible [7]. As seen from the real axis, the process then manifests itself as extra sharp sub-wrinkles seemingly "emitted" suddenly at $x \simeq \pm \bar{x}(\bmod 2 \pi)$ before travelling to one of the main cusps where they eventually join a main condensation. The $N / n$-dependent shape of such separating trajectories thus controls the fate of "supplementary" poles of whatever origin, initial conditions or forcing; this will be exploited elsewhere, though one can already confirm that stable 2-crest steady patterns with $n=O(N) \sim 1 / \nu$ exist if Neumann conditions are employed. With $2 \pi$-periodic conditions these are unstable even if $2 \nu(N+n)=1$, as is seen by considering initial conditions where the $2 n$ poles are slightly shifted to the left of $x=\pi(\bmod 2 \pi)$ : both crests will ultimately merge.

Comparisons with accurate numerical resolutions of the pole equations (45) (46) are again good, Fig 9, For $\nu=1 / 199.5, N=80, n=20$ they yield $\bar{x}=2.053973$ whereas our prediction (65), with $A$ and $a$ iteratively obtained from the normalization conditions (60) (61), gives $\bar{x}=2.053888$. Like $R(B)$ and $r(b), \phi(x)$ cannot be obtained in closed form, yet is readily accessible numerically. Also, if $A=a$, elementary trigonometry shows that the predicted flame slope (63) resumes the result (35), up to a two-fold reduction in $x$ - and $B_{\max }$-scales. 


\section{CONCLUDING REMARKS \& OPEN PROBLEMS}

The above analyses may convey the feeling that the pole densities obtained so far have a family likeness, which is true because they were all deduced from the solution (16) pertaining to isolated crests via adequate changes of independent variable. Whereas (16) itself basically follows from standard Fourier analysis combined with a lucky re-summation of the series thus obtained (15), it would be interesting to understand why the changes of variable (27) then (56) work so well. Admittedly the integral equation (26) bears some formal resemblance with (7), which guided us to propose the new variable (27); but that introduced in (56) looks more strange to us, and was actually discovered by trial-and-error after the 'resolvent' integral equation (55) is obtained. Yet (56) unlikely solely comes "out of the blue". In effect, one may note that (56) is equivalent to $\tanh (\beta)=\tanh \left(\beta_{\max }\right) \sin \theta$ if one defines $\tanh ^{2}\left(\beta_{\max }\right) \equiv \tanh \left(B_{\max } / 2\right) \tanh \left(b_{\max } / 2\right)$ and $\operatorname{sets} \tanh (\beta / 2)=\tanh \left(\beta_{\max } / 2\right) \sin \sigma$, which clearly mirrors what was employed to map the monocoalesced periodic case onto the isolatedcrest problem. Hence (56) rests on the celebrated composition law for hyperbolic tangents $\left(\tau_{1}, \tau_{2}\right): \tau_{1} * \tau_{2}=\left(\tau_{1}+\tau_{2}\right) /\left(1+\tau_{1} \tau_{2}\right)$. It would be interesting to know whether the associated group properties give access to still more general solutions to the Sivashinsky equation (1). That the scale-invariant signum function featured in (7) (8) is left unchanged by the successive changes of variables also is a key property that traces back to the presence of the Hilbert transform $\hat{H}\left(-\phi_{x}\right)$ in (11): in fine, it expresses that the complex velocity about the flame is a sectionally-analytic function in the complex $x$-plane, which is indeed a robust statement for it is little affected by conformal changes of variables that would leave the real axis globally invariant.

Normalizing $P(B)$ to $2 N$ brings about the grouping $N \nu$ and, as long as the integral equations (7) (8) of the continuous approximation are concerned, there is no reason why $N$ should be an integer. Thus, (7) (8) effectively admit a 1-parameter continuum of solutions. The situation is - in a sense, analogous to the Saffman-Taylor problem of viscous fingering and related ones (see [20] and the references therein): when surface effects (here curvature) are omitted, a continuum of steady patterns is found. The equation (11) for flames is peculiar, however, because one knows from the very beginning that only a discrete set of steady monocoalesced solutions exist, corresponding to $N$ s that are integers less than a well-defined $\nu$-dependent value, $N_{\text {opt }}(\nu)$. The Sivashinsky equation (1) thus offers the opportunity to see 
how the WKB approaches to finger-width selection developed [20] for the Saffman-Taylor problem, or kin, can be transposed to the present system to obtain a quantization condition on $N \nu$; for here "inviscid" solutions are now available and one knows the answer in advance. This analysis likely is a key step to study flame response to noise, but has not yet been completed. Because WKB approaches essentially look for solutions of a linearised equation in the form $\exp \left(i \int^{x} k\left(x^{\prime}\right) d x^{\prime}\right)$, where $k(x)=O(1 / \nu)$ depends on the "inviscid" solution, it is seen that obtaining the latter to leading $(O(1))$ order in $\nu$ is not enough. Hence the question: how to compute the leading $(O(\nu)$ ?) correction to the flame profiles obtained above? Obviously this would require to better understand the nature of the continuous approximation leading to the integral equations (7)-(9) or (47) (48) for pole densities. In this context one may perhaps adopt the - rather unusual - view point that the exact pole equations (5), once specialized to $z_{\alpha}=i B_{\alpha}$ and steady patterns, constitute Gauss-like quadrature formulae to evaluate (17) numerically. How to define a "best" way of choosing the pivotal values, i.e. the $B_{\alpha}$ s, naturally leads [21] to the notion of orthogonal polynomials associated with the Sivashinsky equation (1). In the case of Wigner's equation (11) the Hermite polynomials are invoked [14], but we are not aware of such mathematical analyses about (11) and (7) (8).

Next, we recall that 2-crested patterns studied in Sec.VII also belong to a continuous family of solution profiles, now indexed by two independent parameters $N \nu, n \nu$. Even if $N+n$ is assumed to be given by the optimum value $N_{\text {opt }}(\nu) \simeq 1 / 2 \nu$, there still remains the question of how $N / n$ is selected in numerical resolutions of (1) with Neumann conditions at $x=0, \pi$. The ratio $N / n$ can undoubtedly be chosen by the initial flame shape $\phi(x, 0)$. In the case of forced propagations, the noise intensity $(\mu)$ might well play also a role, for one can imagine situations where $\exp (-\pi / 2 \nu) \ll \mu \ll \exp (-\pi / 4 \nu)$ : the noise is then intense enough to break monocoalesced patterns (see Introduction), yet too weak to noticeably affect the more curved 2-crested patterns with $N=n$.

To tailor a global criterion as to compare the 2-crest patterns and their response to noise, the following remarks could be of some use. Let us collectively denote the $B_{\alpha}$ s and $b_{\gamma}$ s as $\boldsymbol{B}$ and $\boldsymbol{b}$, respectively. The unsteady versions of (45) (46) - the pole equations for bicoalesced patterns - may be re-written as

$$
\frac{d \boldsymbol{B}}{d t^{\prime}}=-\nabla_{\boldsymbol{B}} U, \quad \frac{d \boldsymbol{b}}{d t^{\prime}}=-\nabla_{\boldsymbol{b}} U
$$


in terms of $U(\boldsymbol{B}, \boldsymbol{b})=V(\boldsymbol{B})+v(\boldsymbol{b})+w(\boldsymbol{B}, \boldsymbol{b})$, with

$$
\begin{aligned}
V(\boldsymbol{B}) & =\nu \sum_{\alpha}\left|B_{\alpha}\right|-2 \nu^{2} \sum_{\alpha, \beta<\alpha} \log \left|\sinh \frac{B_{\alpha}-B_{\beta}}{2}\right|, \\
w(\boldsymbol{B}, \boldsymbol{b}) & =-2 \nu^{2} \sum_{\gamma, \beta} \log \left(\cosh \frac{b_{\gamma}-B_{\beta}}{2}\right),
\end{aligned}
$$

and an expression similar to (67) for $v(\boldsymbol{b}) ; t^{\prime}=t / \nu$ is time scaled by the shortest growth time of small-scale wrinkles (see Introduction). Accordingly, when the right-hand sides of (66) are supplemented with statistically identical, independent random (e.g., Gaussian) additive forcings, the joint probability density of $(\boldsymbol{B}, \boldsymbol{b})$ will tend to a quantity $\sim \exp \left(-U(\boldsymbol{B}, \boldsymbol{b}) / \mu^{2}\right)$, where $\mu \ll 1$ characterizes the noise intensities. Because $U \sim 1$ in the small- $\nu$ limit (since $P(B)$ and $p(b)$ are $O(1 / \nu))$ the above exponential is strongly peaked about the steady solutions. One can thus think of employing the $N / n$-dependent scalar $U(\boldsymbol{B}, \boldsymbol{b})$, evaluated at steady state, as an objective means to discriminate the various 2-crested patterns in the presence of forcing. The task of evaluating $U$ in the continuous approximation has not yet been completed. Neither is the analysis required to handle situations where the poles are slightly misaligned... yet still symmetric about $x=0$ and $x=\pi$ for compatibility with Neumann boundary conditions.

One must finally stress that the present analyses did not exhaust all the possibilities of "steady" solutions of (1), even with $2 \pi$ as minimal periodicity. The interpolating solutions discovered in [7, 22] constitute another class, comprising (possibly many-) extra poles, nearly evenly distributed [17, 18] along sinuous curves at a distance from the real axis. In our opinion such unstable equilibriums are also worth analyzing in detail for $\nu \rightarrow 0$, as are those mentioned in Sec VI and generalizations of (11) itself [23].

As an end to a numerical work on (1), with noise included in the right hand side [7], one of us concluded that "... it is likely that new analytical studies of the Sivashinsky equation should be possible: even if the equation is now almost 30 years old, many things remain to be explained". The words still hold true. 


\section{Acknowledgments}

One of us (G.J.) thanks H. El-Rabii (Poitiers University) for discussions, helps in the Calculus... and the $\mathrm{LTE}_{\mathrm{E} X}$ version of the paper.

[1] L. D. Landau, Acta Physicochimica USSR 19, 77 (1944).

[2] G. Darrieus (1938), unpublished work presented at La Technique Moderne, Paris.

[3] G. I. Sivashinsky, Acta Astron. 4, 1177 (1977).

[4] K. A. Kazakov, Phys. Fluids 17, 032107 (2005).

[5] G. I. Sivashinsky and P. Clavin, J. Phys. (France) 48, 193 (1987).

[6] D. N. Michelson and G. I. Sivashinsky, Acta Astron. 4, 1207 (1977).

[7] B. Denet, Phys. Rev. E 74, 036303 (2006).

[8] G. Joulin, J. Phys. (France) 50, 1069 (1989).

[9] B. Denet, Phys. Rev. E 75, 046310 (2007).

[10] O. Thual, U. Frisch, and M. Hénon, J. Phys. (France) 46 (1985).

[11] Y. Lee and H. Chen, Phys. Scr. 2, 41 (1982).

[12] L. Landau and E. Lifschitz, Fluid Mechanics (Pergamon Press, Oxford, 1979).

[13] G. K. Batchelor, An introduction to fluid dynamics (Cambridge University Press, Cambridge, 1967).

[14] M. L. Mehta, Random Matrices (Academic Press, Boston, 1991), 2nd ed.

[15] I. S. Gradshteyn and I. M. Ryzhik, Table of Integrals, Series and Products (Elsevier-Academic Press, Amsterdam, 2007), 7th ed.

[16] G. Joulin, Combust. Sci. Techn. 53, 315 (1987).

[17] D. Vaynblatt and M. Matalon, SIAM J. Appl. Math. 60, 703 (2000).

[18] O. Kupervasser, Z. Olami, and I. Procaccia, Phys. Rev. E 59, 2587 (1999).

[19] J. Bec, U. Frisch, and K. Khanin, J. Fluid Mech. 416, 239 (2000).

[20] P. Pelcé, Théorie des formes de croissance (EDP Sciences - CNRS Edition, Paris, 2000).

[21] G. E. Andrews, R. Askey, and R. Roy, Special Functions (Cambridge University Press, Cambridge, 2000).

[22] L. F. Guidi and D. H. U. Marchetti, Phys. Lett. A 308, 162 (2003). 
[23] G. Joulin, Zh. Eksp. Teor. Fiz. 100, 428 (1991). 


\section{List of figures}

Fig. 1: Numerical vs analytical cumulative pole densities, for an isolated crest with $1 / \nu=19.5, N=10$. If exact, the theoretical curve (dot-dashed line, eq. (18)) would pass through the middle of the risers of the numerical staircase (solid line, eq. (5) ). The dashed

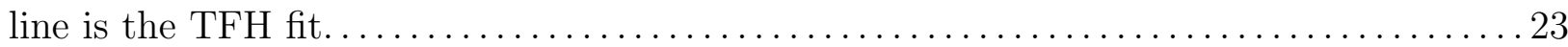

Fig. 2: Same as in Fig. 1, for $1 / \nu=199.5, N=100$. Only the upper hull (solid line) of the exact staircase is shown, for readability. The dashed line is the TFH fit. ... 24

Fig. 3: Numerical ((20), solid line) vs analytical ((17), dot-dashed line) pole densities $P(B)$ for an isolated crest with $1 / \nu=199.5, N=100$. The dashed line is the TFH

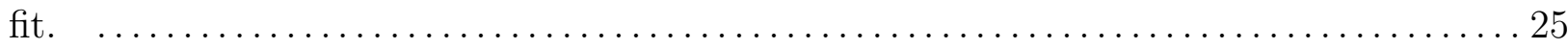

Fig. 4: Shapes of an isolated crest with $1 / \nu=19.5, N=10$ : continuous approximation ((25), dot-dashed line), exact (solid line), and smooth approximation from eq. (22)

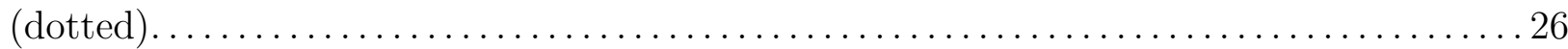

Fig. 5: Numerical (solid line) vs analytical (eq. (29), dot-dashed line) cumulative pole densities for a monocoalesced periodic crest, for $1 / \nu=199.5$, and $N=100$ $\left(=N_{\text {opt }}(\nu)\right)$. Only the upper hull of the numerical staircase is shown $\ldots \ldots \ldots \ldots \ldots \ldots 27$

Fig. 6: Shapes of a monocoalesced periodic flame with $1 / \nu=19.5, N=10\left(=N_{\text {opt }}(\nu)\right)$ :

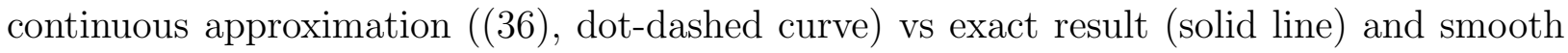

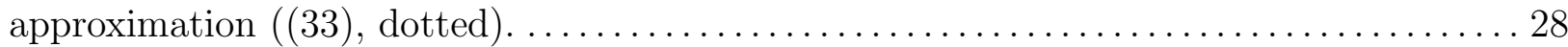

Fig. 7: Cumulative pole densities $R(B)$ (upper curves) and $r(b)$ for a bicoalesced periodic pattern with $1 / \nu=600.5, N=200, n=100$ : the solid and the dotted lines are from eqs. (53) (154) and (57) (56); the dashed and the dot-dashed ones are the upper hulls of the exact staircases (see Fig. 1). As $(N+n)=N_{\text {opt }}(\nu), B_{200}=\infty \ldots \ldots \ldots \ldots \ldots \ldots$

Fig. 8: Theoretical pole densities $P(B)$ (resp. $p(b)$ ) plotted as dot-dashed or dashed 
lines vs $\theta$, eq. (57), with $\sin \sigma$ replaced by $(\tanh B / 2) / A($ resp. $(\tanh b / 2) / a)$. The solid and the dotted lines are the numerical pole densities. All are for a bicoalesced periodic

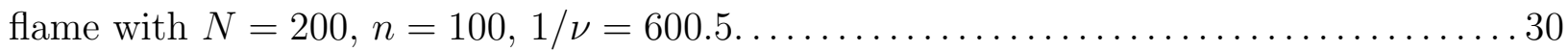

Fig. 9: Shapes of a bicoalesced flame with $1 / \nu=199.5, N=80, n=20$ : exact (solid line) vs continuous approximation (from integration of (63), dot-dashed).........3 31 


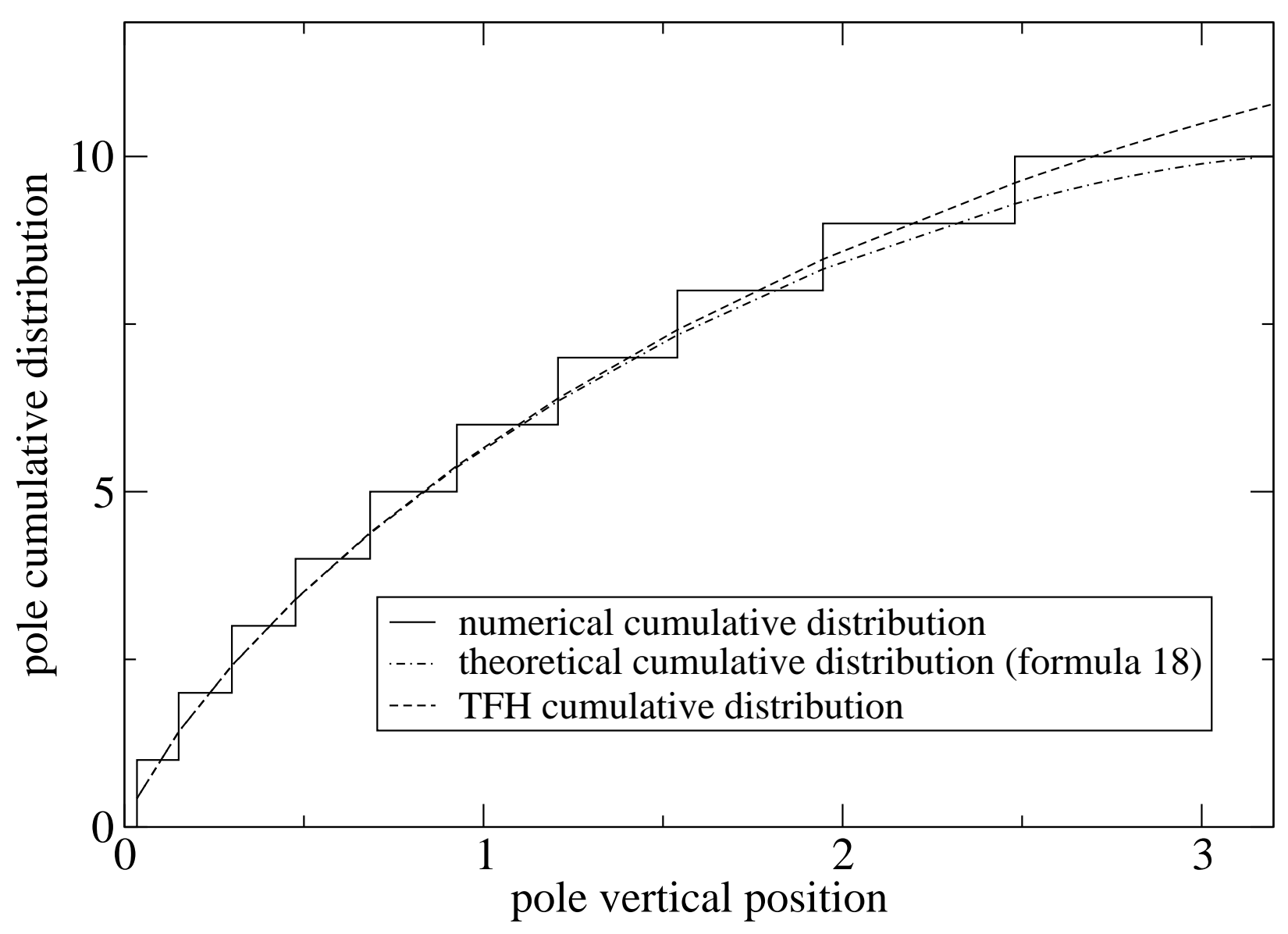

FIG. 1: Numerical vs analytical cumulative pole densities, for an isolated crest with $1 / \nu=19.5$, $N=10$. If exact, the theoretical curve (dot-dashed line, eq. (18)) would pass through the middle of the risers of the numerical staircase (solid line, eq. (5)). The dashed line is the TFH fit. 


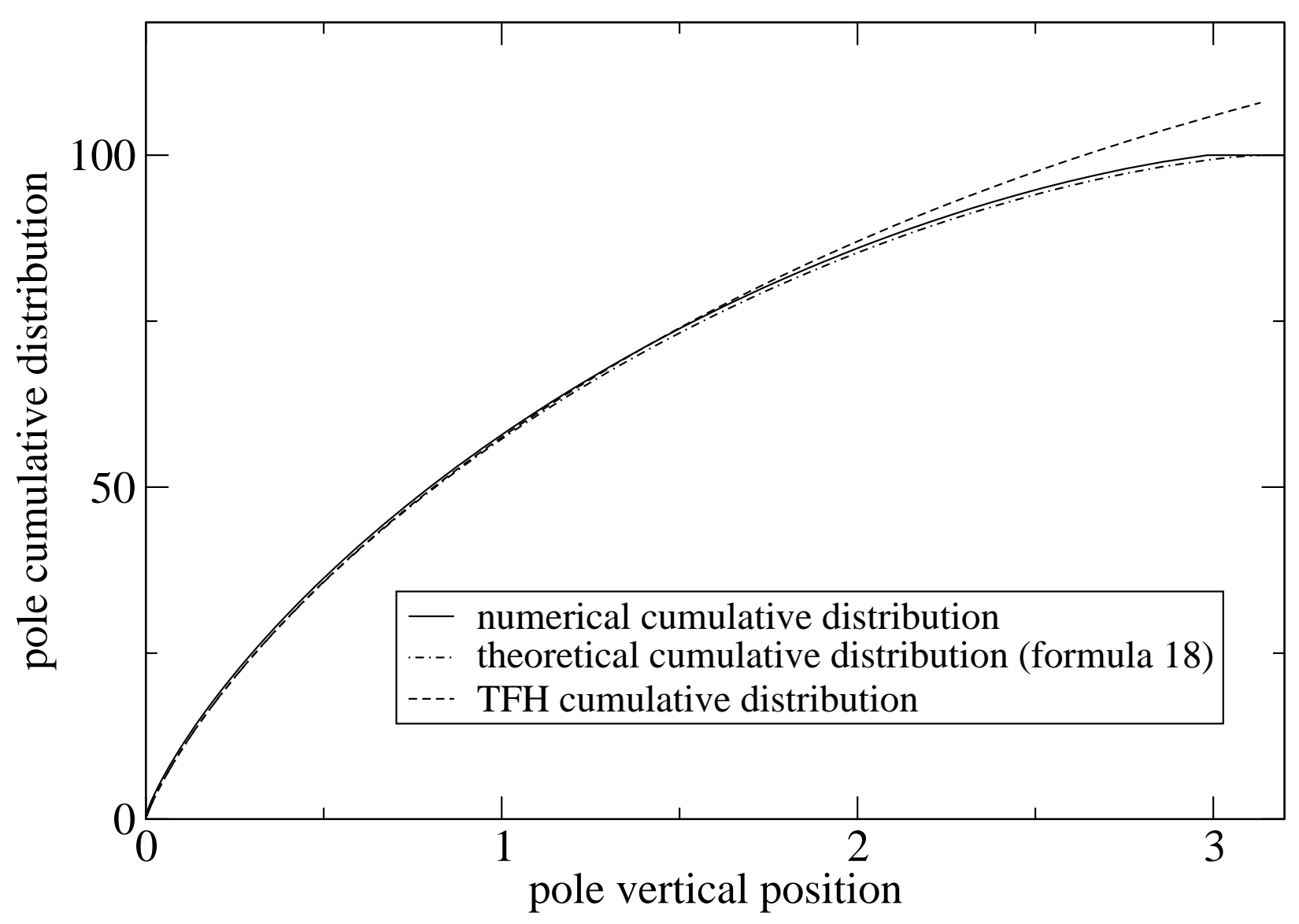

FIG. 2: Same as in Fig. 1, for $1 / \nu=199.5, N=100$. Only the upper hull (solid line) of the exact staircase is shown, for readability. The dashed line is the TFH fit. 


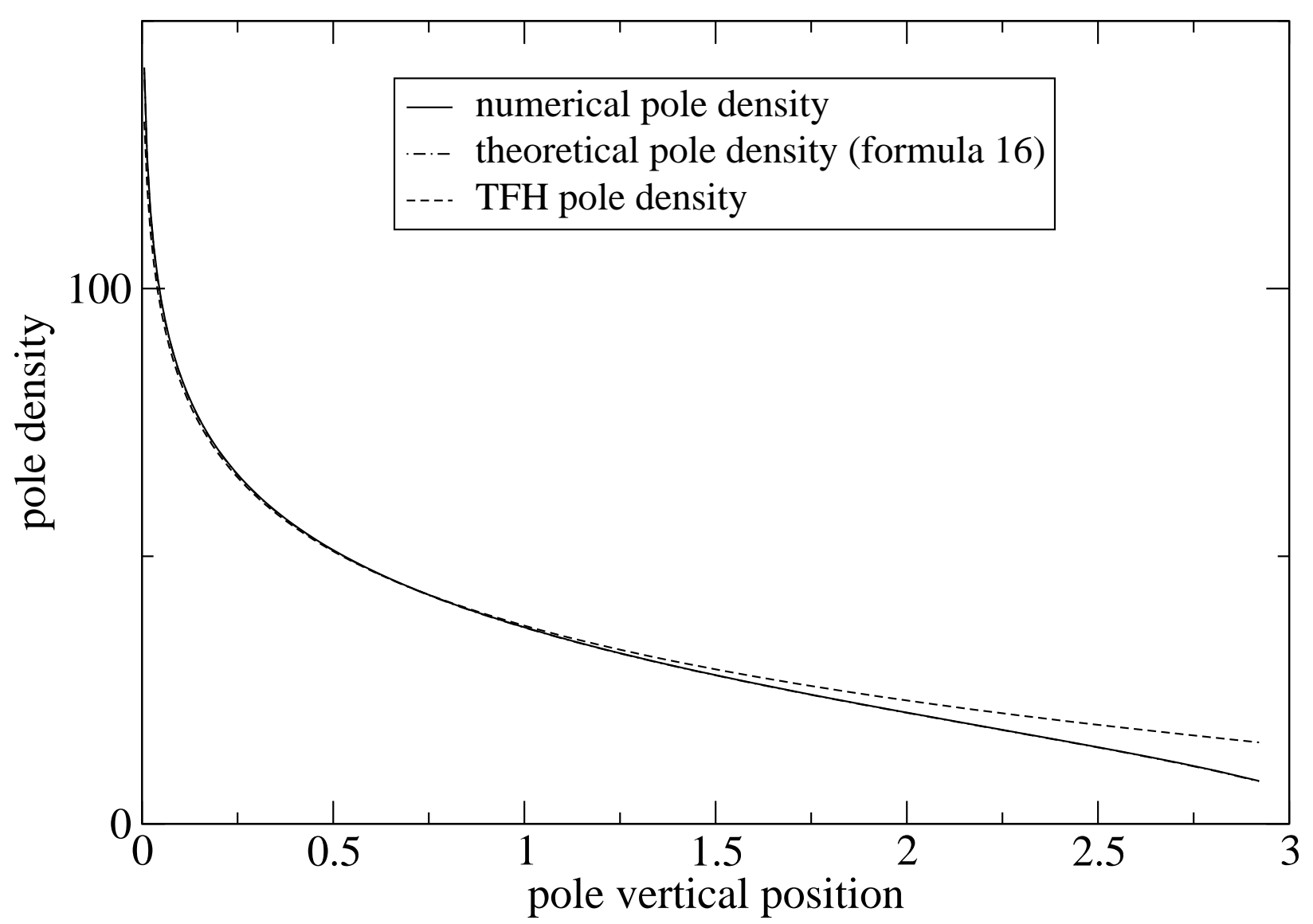

FIG. 3: Numerical ((20), solid line) vs analytical ((17), dot-dashed line) pole densities $P(B)$ for an isolated crest with $1 / \nu=199.5, N=100$. The dashed line is the TFH fit. 


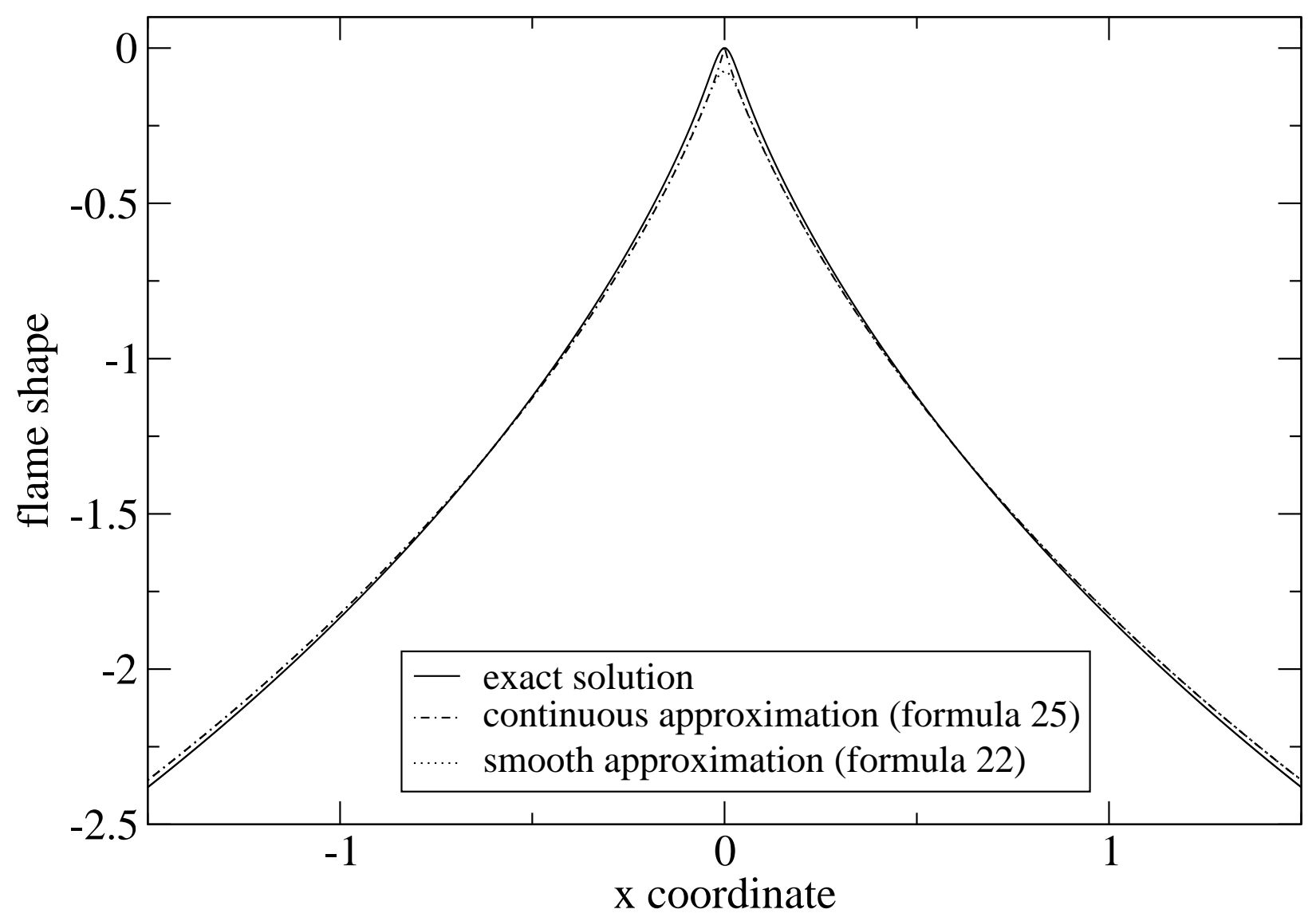

FIG. 4: Shapes of an isolated crest with $1 / \nu=19.5, N=10$ : continuous approximation ((25)), dot-dashed line), exact (solid line), and smooth approximation from eq. (22) (dotted). 


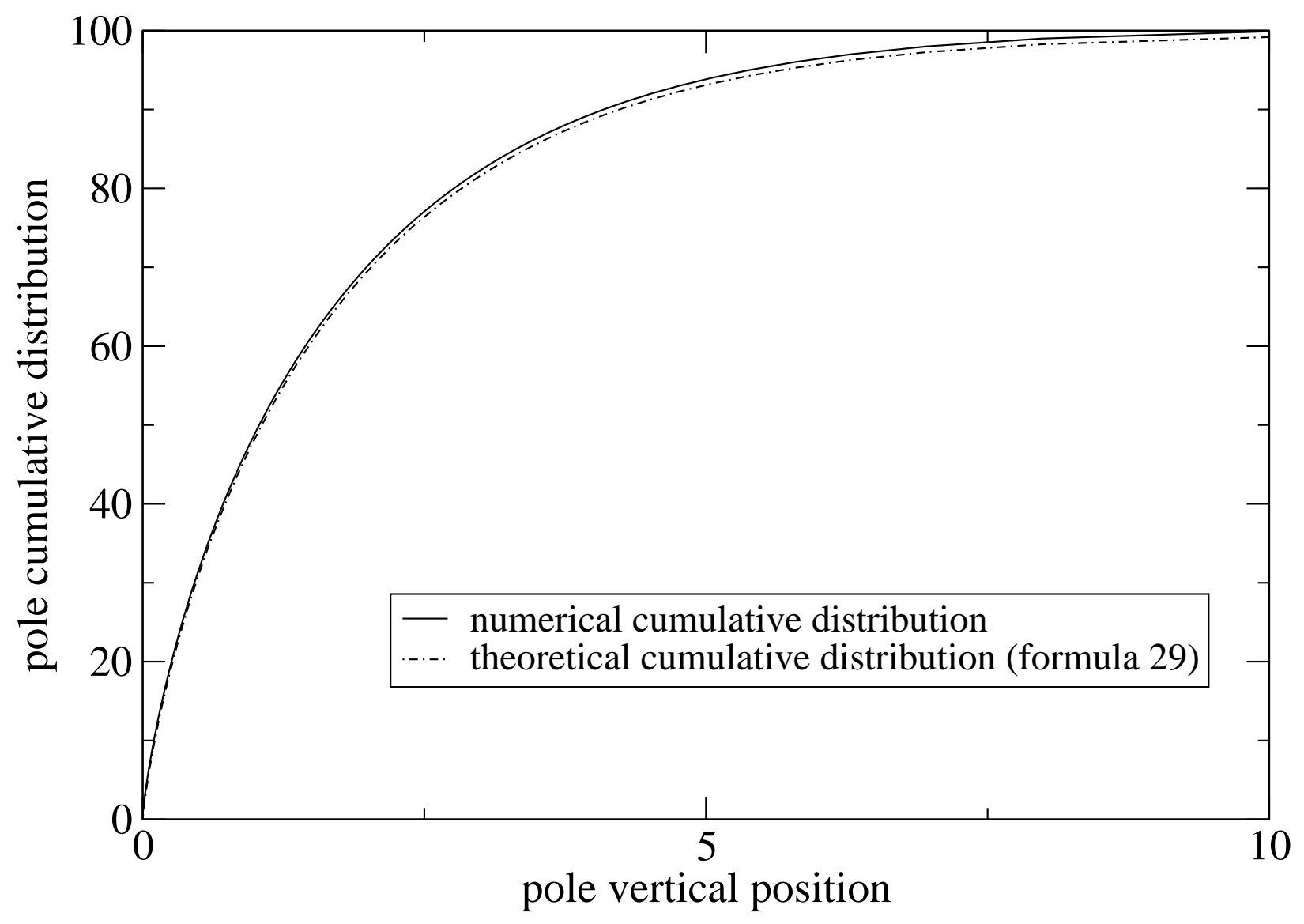

FIG. 5: Numerical (solid line) vs analytical (eq. (29), dot-dashed line) cumulative pole densities for a monocoalesced periodic crest, for $1 / \nu=199.5$, and $N=100\left(=N_{\text {opt }}(\nu)\right)$. Only the upper hull of the numerical staircase is shown. 


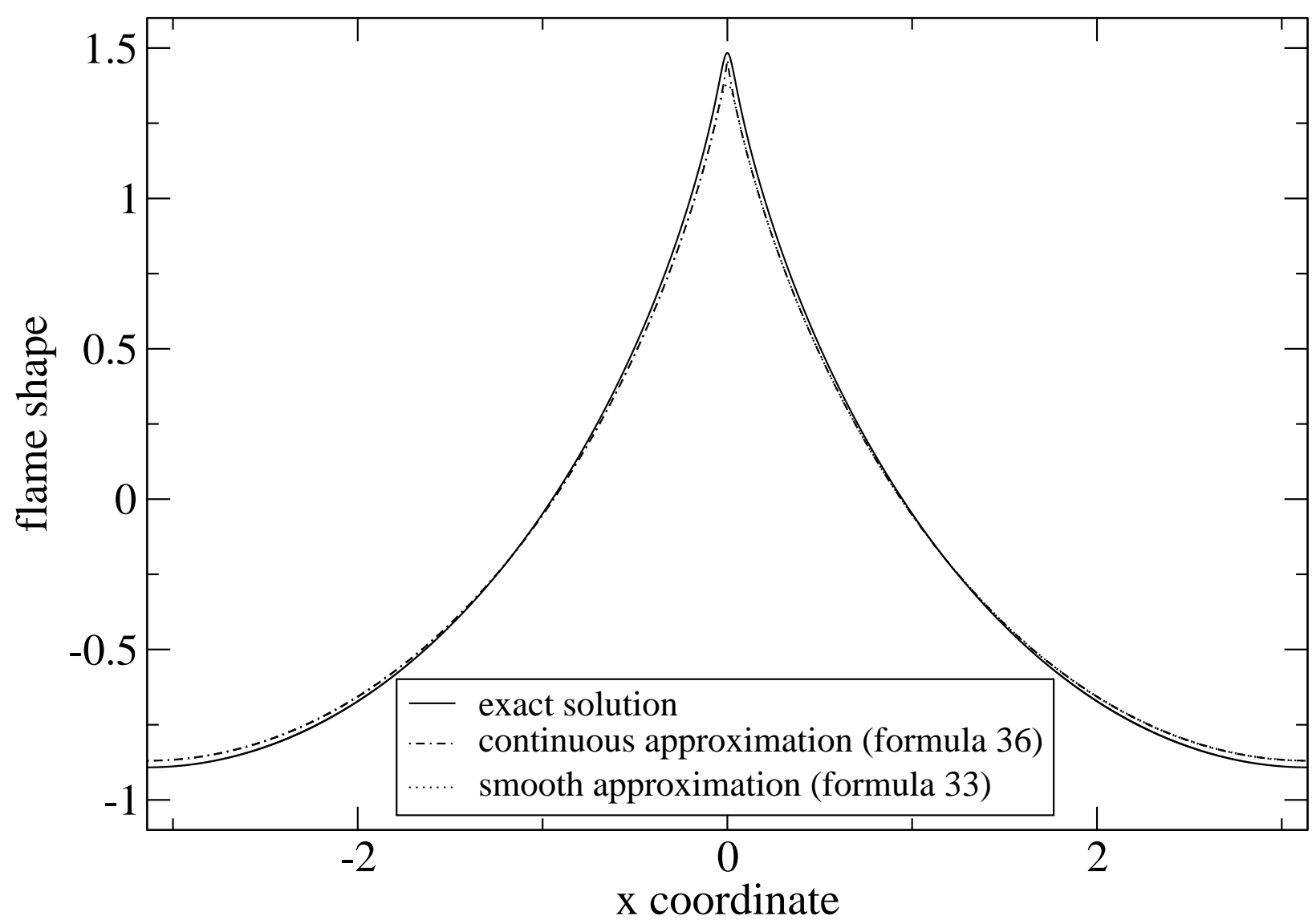

FIG. 6: Shapes of a monocoalesced periodic flame with $1 / \nu=19.5, N=10\left(=N_{\text {opt }}(\nu)\right)$ : continuous approximation ((36) $)$, dot-dashed curve) vs exact result (solid line) and smooth approximation (([33), dotted). 


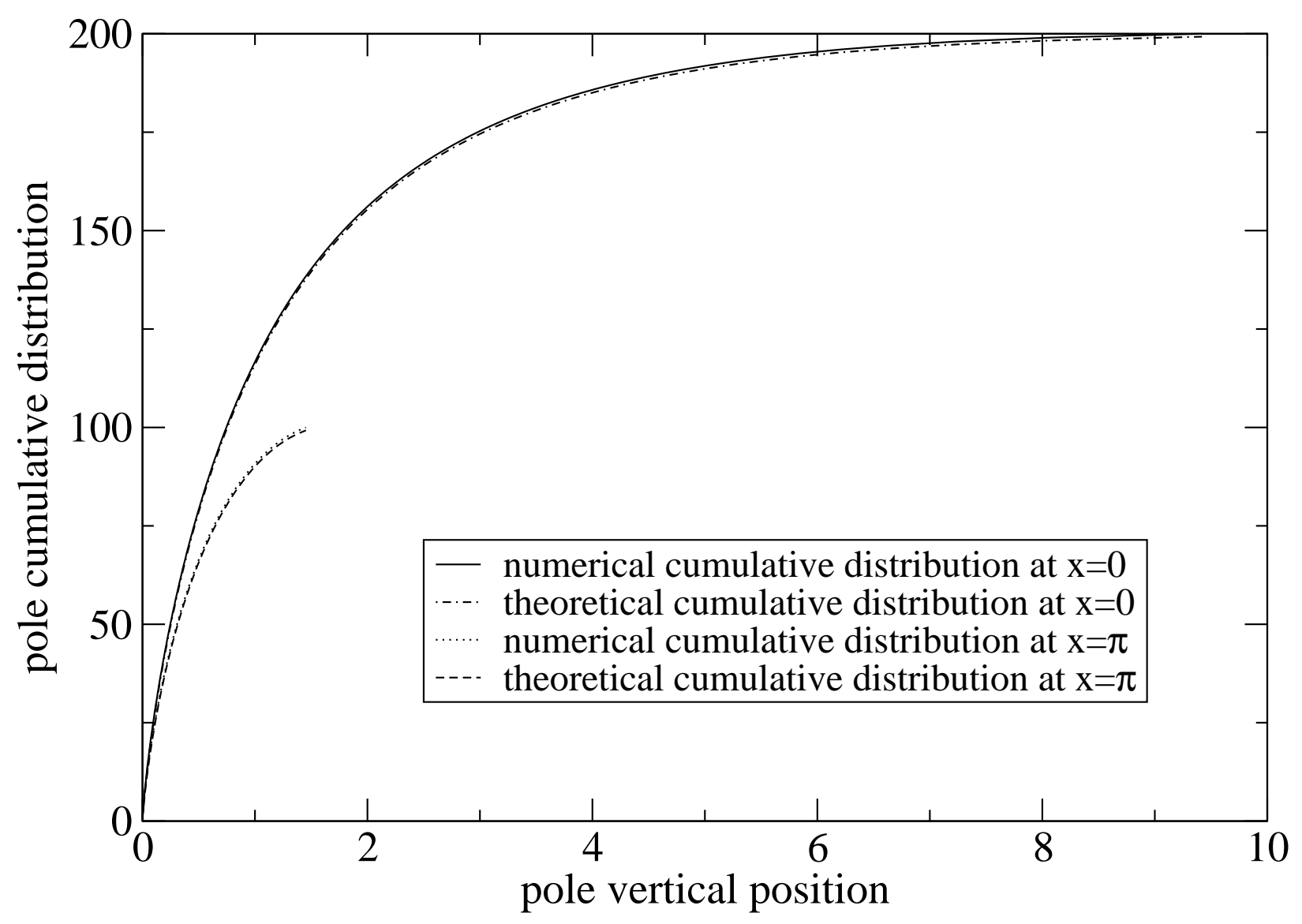

FIG. 7: Cumulative pole densities $R(B)$ (upper curves) and $r(b)$ for a bicoalesced periodic pattern with $1 / \nu=600.5, N=200, n=100$ : the solid and the dotted lines are from eqs. (53) (54) and (57) (56); the dashed and the dot-dashed ones are the upper hulls of the exact staircases (see Fig.

1). As $(N+n)=N_{\text {opt }}(\nu), B_{200}=\infty$. 


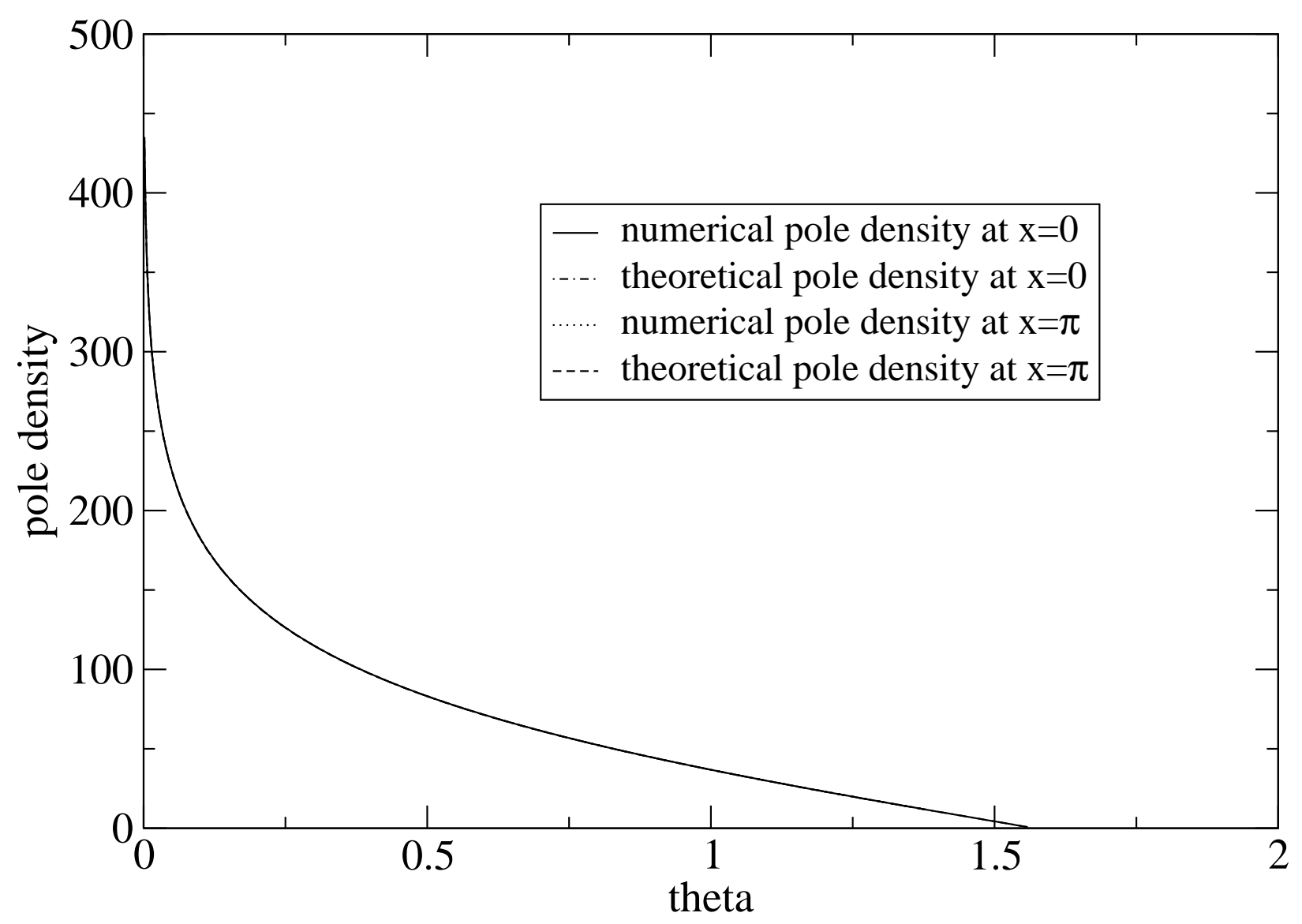

FIG. 8: Theoretical pole densities $P(B)$ (resp. $p(b)$ ) plotted as dot-dashed or dashed lines vs $\theta$, eq. (57), with $\sin \sigma$ replaced by $(\tanh B / 2) / A(\operatorname{resp} .(\tanh b / 2) / a)$. The solid and the dotted lines are the numerical pole densities. All are for a bicoalesced periodic flame with $N=200, n=100$, $1 / \nu=600.5$. 


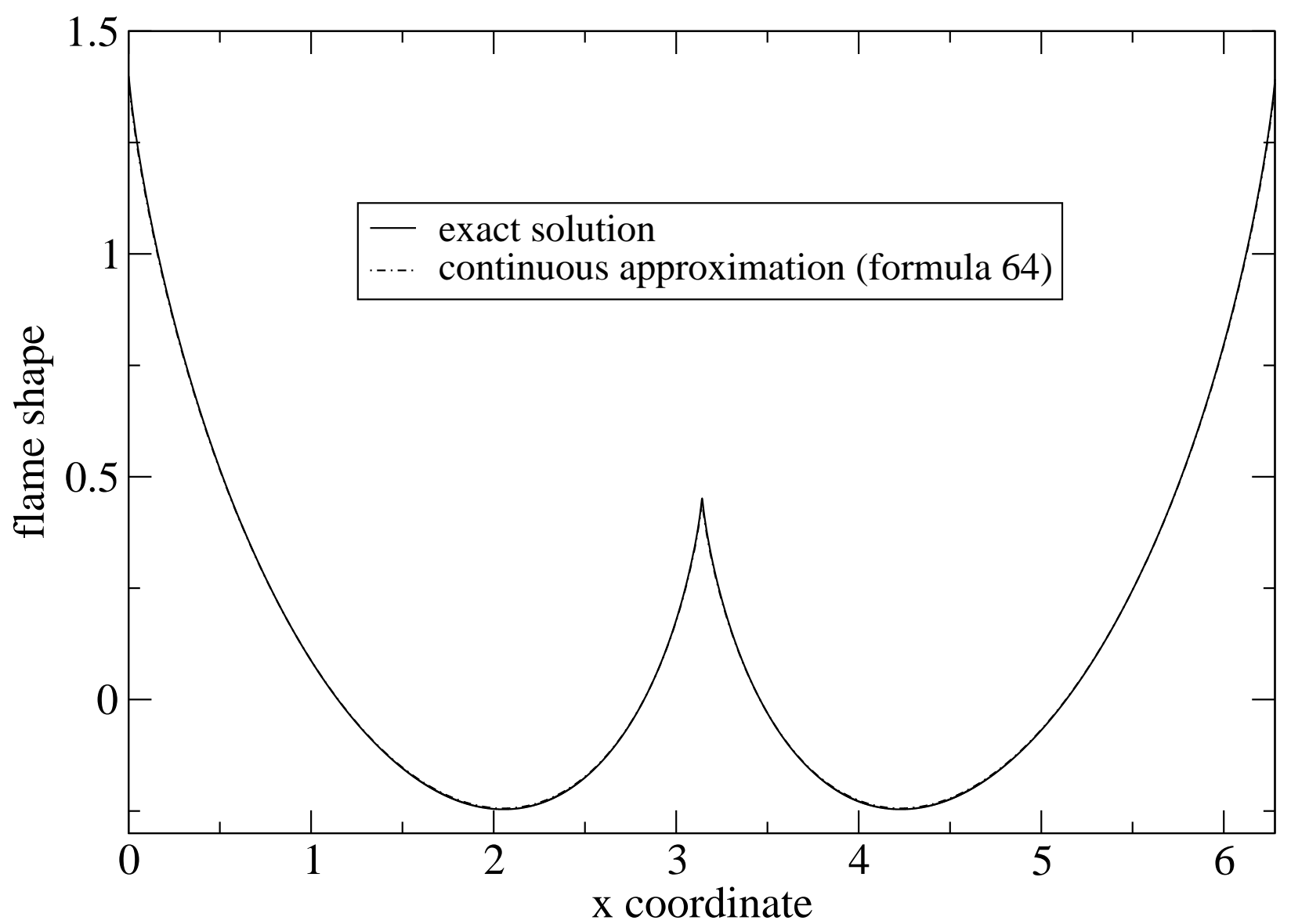

FIG. 9: Shapes of a bicoalesced flame with $1 / \nu=199.5, N=80, n=20$ : exact (solid line) vs continuous approximation (from integration of (63), dot-dashed). 